\title{
Revision of the genus complex Gibbula: an integrative approach to delineating the Eastern Mediterranean genera Gibbula Risso, 1826, Steromphala Gray, 1847, and Phorcus Risso, 1826 using DNA-barcoding and geometric morphometrics (Vetigastropoda, Trochoidea)
}

\author{
Susanne Affenzeller ${ }^{1,2}$ • Nicole Haar ${ }^{1}$ • Gerhard Steiner ${ }^{1}$ (D)
}

Received: 19 December 2016/Accepted: 5 September 2017 /Published online: 7 October 2017

(C) The Author(s) 2017. This article is an open access publication

\begin{abstract}
The trochoid genus, Gibbula, is abundant and diverse in the Mediterranean Sea but problematic to identify and delineate. This is due to highly variable shell morphology, vague original descriptions, and missing or unspecific type material. In recent studies, COI barcoding yielded satisfactory results for species delineation. In the present study, a combination of geometric shell morphometric methods and COI barcoding was used to assess the most abundant species of the Eastern Mediterranean. All relevant identification characters were captured via standardised images of the shells in both lateral and ventral views. Agreeing with previous studies, Gibbula was recovered as paraphyletic in the molecular analysis and thus is restricted to the clade encompassing the type species Gibbula magus (Linnaeus, 1758). The geometric morphometric analyses and the barcoding approach clearly distinguish the remaining species into two groups: the genus Steromphala Gray, 1847 and the genus Phorcus Risso, 1826. Type material was used for the geometric morphometric analyses whenever possible. Based on re-examination of the original type descriptions, lectotypes were designated. The joint application of DNA-barcoding and geometric
\end{abstract}

Electronic supplementary material The online version of this article (https://doi.org/10.1007/s13127-017-0343-5) contains supplementary material, which is available to authorized users.

Gerhard Steiner

gerhard.steiner@univie.ac.at

1 Department of Integrative Zoology, University of Vienna, Althanstraße 14, 1090 Vienna, Austria

2 Department of Geobiology, Georg-August-University Göttingen, Goldschmidtstraße 3, 37077 Göttingen, Germany morphometrics not only effectively delineated the sister genera Steromphala and Phorcus but also delineated all analysed species in the Gibbula-Steromphala-Phorcus genus complex. The additional use of geometric morphometrics enables researchers to compare barcoded material with fossil specimens or dry collections in an objective way.

Keywords Gibbula Phorcus · Steromphala · Barcoding · Geometric morphometrics $\cdot$ Species delineation

\section{Introduction}

Gastropod taxonomy is traditionally shell-based. Methodology in recent years using genetic data has revealed cryptic species and questionable species delineation in several gastropod taxa (e.g. Delicado and Ramos 2012; Weigand et al. 2013). Therefore, species identification and delineation by shell morphology alone is often mistrusted if not supported by additional lines of evidence, such as DNA-barcoding or morphometric data. However, external characteristics (e.g. shell characters) remain the most straight forward way of identification of snails by scientists from related fields such as ecology or palaeontology and especially by amateurs. Reliable species identifications and delineations of even cryptic lineages not only are significant for taxonomic purposes but also impact the assessment of biodiversity, ecological niche differentiation, and conservation measurements (e.g. Bálint et al. 2011; Feckler et al. 2014). Thus, it is of paramount importance-particularly for common taxa-to re-evaluate the usefulness of shell morphology for species delineation and identification, based on independent molecular markers. Mediterranean trochid 
gastropods provide a suitable platform for re-evaluating earlier hazy species descriptions and delineations in a contemporary context.

The systematics and phylogenetic relationships of the trochoid families Trochidae and Turbinidae and the subfamily Cantharidinae are only partly resolved (Williams and Ozawa 2006; Williams et al. 2008, 2010). Although molecular data support the monophyly of the Cantharidinae (Williams et al. 2010; Uribe et al. 2016), relationships within the subfamily remain ambiguous.

One of the subfamily's most problematic groups contains Gibbula Risso, 1826 and Phorcus Risso, 1826. Problems with correctly identifying Gibbula and Phorcus species are main reasons for this unresolved status. An umbilicated shell, black and white striped epipodial tentacles, a non-calcified operculum, and a turbinate shell form currently characterise both genera. Species differ in spiral height, umbilicus size and shape, and overall shell form. Shell sculpture is diagnostic for a few species, and even coloration is sometimes used in the literature. However, all these characters show such a wide range of variation that reliable identification of species to Gibbula and Phorcus is difficult and depends on subjective, personal experience (e.g. Barco et al. 2013).

Among the reasons for the confusing status of some of these species are their vague original descriptions, mostly based on dry and empty shells. To exacerbate the situation, some of the type collections have been destroyed or lost (Table 1), making re-descriptions impossible. Other type collections contain many syntypes, some of which do not show consistent morphology. The vagueness of original descriptions leads to problems in recent literature used for identification, e.g. for the identification of G. varia, G. rarilineata and juvenile G. divaricata (Gofas et al. 2011; Nordsieck 1968), as well as G. umbilicaris and G. nebulosa (Barco et al. 2013; Oliverio pers. comm.).

In the comprehensive phylogenetic study by Donald et al. (2012), the monophyly of Gibbula, represented by G. magus, G. fanulum, G. cineraria, G. pennanti, G. rarilineata, G. umbilicalis, and G. varia, is disrupted by species of Osilinus, a junior synonym of Phorcus (Donald et al. 2012), and Williams et al. (2010) by Jujubinus (See Table 1 for taxon authorities). In the most recent molecular study based on six different genes (both mitochondrial and nuclear) by Uribe et al. (2016), the monophyly of Gibbula is disrupted by both Phorcus and Jujubinus. In the most comprehensive molecular study on the genus Gibbula, Barco et al. (2013) resolved the dilemma by using Phorcus species as outgroup and omitted other cantharidinid genera such as Jujubinus, whereby Gibbula was recovered monophyletic.

The morphological and phylogenetic problems in Gibbula and Phorcus (Philippi 1836-44, 1849; Thiele 1929-35; Nordsieck 1968) and their taxonomic implications were addressed by different authors (e.g. Williams et al. 2010, Uribe et al. 2016) introducing several subgenera (e.g. Steromphala Gray, 1847, Phorcus Risso, 1826, Gibbulastra Monterosato, 1884) which later were discarded or revised again. Risso's (1826) original species allocations to the two genera, Gibbula and Phorcus, were later re-established (Thiele 1929-35; Nordsieck 1968; Gofas and Jabaud 1997). Phylogenetic and systematic relationships remain unresolved.

\section{Shell morphometrics}

Morphometric methods have been successful in identifying certain species of gastropods via analysis of shell form (Kirchner et al. 2016). Since the classical morphometric measures like lengths and angles introduced by Thompson (1917) are insufficient to capture shape differences in the present taxa, geometric morphometric approaches utilising landmark-based algorithms are a valuable alternative. Mathematically, they are increasingly applied to assess form and shape as well as variation and intended as an objective means of comparison (Rohlf 1998). Landmarks and semi-landmarks offer the possibility to analyse objects without losing their geometrical form in the process (Bookstein 1991; Mitteroecker and Gunz 2009). Gastropod shells offer only few useful morphological landmarks as defined by Bookstein (1991). Thus, it is necessary to use semi-landmarks on outlines, which are processed later on in the sliding landmark algorithm, allowing for the landmark to slide on a tangent connection between neighbouring landmarks in order to optimise the Procrustes fit (Bookstein 1997; Mitteroecker and Gunz 2009). Cartesian coordinates gained from landmarks and semi-landmarks are aligned in a generalised Procrustes fit. Through a stepwise process, the original landmark configuration of each individual is scaled and rotated in a way to gain a group of landmark configurations showing the least possible difference. In this process, each resulting landmark configuration therefore, independent of size, orientation, and position, only comprises shape in the end (Bookstein 1996). The resulting Procrustes coordinates define the shape of an object in the Kendall shape space (Kendall 1981, 1984). Although its surface is curved, Procrustes distances can be approximated by Euclidean distances in the tangent space and can thus be analysed statistically through multivariate methods like principal components analyses (Mitteroecker and Gunz 2009). Geometric morphometrics, thus, offers the possibility of analysing and comparing complex shapes of shells of different sizes in a single sample (Mitteroecker and Gunz 2009).

\section{Aims of the study}

The genera Gibbula and Phorcus have undergone considerable changes in their 190 years of history. The rather vague morphological diagnosis of Gibbula and Phorcus left ample room for species assignment to these taxa (compare Nordsieck 
Table 1 Authorities, type localities, and location of type material of Gibbula (s.s.), Steromphala, and Phorcus species most abundantly found in the Mediterranean

\begin{tabular}{|c|c|c|c|}
\hline Species & Authority & $\begin{array}{l}\text { Collection } \\
\text { (Coll. numbers) }\end{array}$ & Type locality \\
\hline Gibbula ardens & (Salis, 1793) & $?$ & Naples \\
\hline Gibbula fanulum & (Gmelin, 1791) & $?$ & $\begin{array}{l}\text { aquis } \\
\text { sernabucensibus }\end{array}$ \\
\hline Gibbula magus & (Linnaeus, 1758) & $\begin{array}{l}\text { LSL+ZMUU } \\
(\text { LSL.500+UPSZTY 720, 1131) }\end{array}$ & Mare Mediterraneo \\
\hline Gibbula turbinoides & (Deshayes, 1835) & $?$ & fossil, La Moree \\
\hline Gibbula candei & (d'Orbigny, 1844) & $?$ & $?$ \\
\hline Gibbula albida & (Gmelin, 1791) & $?$ & $?$ \\
\hline Gibbula guttadauri & (Philippi, 1836) & $?$ & Aci Castello, Sicily \\
\hline Phorcus articulatus & (Lamarck, 1822) & $\begin{array}{l}\text { MNHG } \\
\text { (INVE 51532) }\end{array}$ & Mediterrané \\
\hline Phorcus mutabilis & (Philippi, 1846) & $?$ & $\begin{array}{l}\text { Adriatic and Ionic } \\
\text { Sea }\end{array}$ \\
\hline Phorcus richardi & (Payraudeau, 1826) & $\begin{array}{l}\text { MNHN } \\
\text { (MNHN-IM-2000-28253) }\end{array}$ & Ile d'Corse \\
\hline Phorcus turbinatus & (Born, 1778) & $\begin{array}{l}\text { MNHW } \\
\text { (NHMW 14002) }\end{array}$ & $?$ \\
\hline Phorcus lineatus & (da Costa, 1778) & $?$ (sold in 1818, London) & British shores \\
\hline Steromphala adansonii & (Payraudeau, 1826) & $\begin{array}{l}\text { MNHN } \\
(\text { MNHN-IM-2000-30071) }\end{array}$ & Golf d'Aiaccio \\
\hline Steromphala adriatica & (Philippi, 1844) & $?$ & Sicily \\
\hline Steromphala divaricata & (Linnaeus, 1758) & $\begin{array}{l}\text { LSL } \\
\text { (LSL.503) }\end{array}$ & Mare Mediterraneo \\
\hline Steromphala nebulosa & (Philippi, 1849) & $?$ & $\begin{array}{l}\text { Alexandria and Red } \\
\text { Sea }\end{array}$ \\
\hline Steromphala rarilineata & (Michaud, 1829) & MHNL & Mediterrané \\
\hline Steromphala spratti & (Forbes, 1844) & $?$ & Aegais \\
\hline Steromphala umbilicaris & (Linnaeus, 1758) & $\begin{array}{l}\text { LSL+ZMUU } \\
\text { (LSL.504+UPSZTY 998, 1127, 1128, } \\
\text { 1130) }\end{array}$ & Mare Mediterraneo \\
\hline Steromphala varia & (Linnaeus, 1758) & $\begin{array}{l}\text { LSL+ZMUU } \\
\text { (LSL.501+UPSZTY 1136) }\end{array}$ & Mare Mediterraneo \\
\hline
\end{tabular}


Table 1 (continued)

\begin{tabular}{|c|c|c|c|}
\hline Steromphala cineraria & (Linnaeus, 1758) & $\begin{array}{l}\text { LSL+ZMUU } \\
\text { (LSL.502+UPSZTY 1134, 1139) }\end{array}$ & Mare Mediterraneo \\
\hline Steromphala nivosa & (A. Adams, 1853) & $\begin{array}{l}\text { NHML } \\
(196898)\end{array}$ & $?$ \\
\hline Steromphala pennanti & (Philippi, 1846) & $?$ & English Coast \\
\hline Steromphala umbilicalis & (da Costa, 1778) & $?$ (sold in 1818, London) & British shores \\
\hline Steromphala leucophaea & (Philippi, 1836) & $?$ & l'Ognina, Sicily \\
\hline Steromphala racketti & (Payraudeau, 1826) & $\begin{array}{l}\text { MNHN } \\
\text { (MNHN-IM-2000-28252) }\end{array}$ & Valinco \\
\hline *Phorcus magaritaceus & Risso 1826 & $\begin{array}{l}\text { MNHN } \\
(\mathrm{MNHN}-\mathrm{IM}-2000-28254,-2000- \\
28255)\end{array}$ & $?$ \\
\hline *Gibbula magus & Risso 1826 & $\begin{array}{l}\text { MNHN } \\
(\mathrm{MNHN}-\mathrm{IM}-2012-36202,-2012- \\
36203,-2012-36204)\end{array}$ & $?$ \\
\hline
\end{tabular}

Asterisks mark collections upon which genus descriptions were based. Species used in this study are shaded. (LSL Linnean Society of London Collection, MHNL Musée des Confluences Lyon, MNHG Museum of Natural History Geneva, MNHN Musée National d'Histoire Naturelle Paris, NHML Natural History Museum London, NHMW Natural History Museum Vienna, ZMUU Zoological Museum University of Uppsala)

1968). As broad taxon sampling in molecular phylogenetic and barcoding studies becomes more frequent, objective identification methods become more important as many of these projects rely on correct species designations based on wellacquired experience of both amateurs and scientists. The present study aims to re-evaluate the delimitation and the relationships of the genus complex Gibbula via an integrative approach based on DNA-barcoding, shell morphometrics, and shell characters using recently collected material with accurate locality data as well as type material of the described taxa. Furthermore, we aim to address questions regarding monophyly and internal systematics of Gibbula and Phorcus raised by recent molecular studies (Donald et al. 2012; Williams 2012; Uribe et al. 2016).

\section{Material and methods}

\section{Sampling}

Snails were collected by snorkelling off the north coast of Crete

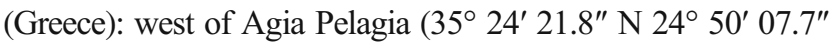
E), south coast: Martsalo valley ( $34^{\circ} 55^{\prime} 48.0^{\prime \prime} \mathrm{N} 24^{\circ} 46^{\prime} 14.0^{\prime \prime}$ E), Dyticus bay ( $\left.34^{\circ} 55^{\prime} 46.2^{\prime \prime} \mathrm{N} 24^{\circ} 54^{\prime} 45.9^{\prime \prime} \mathrm{E}\right)$, Lentas ( $34^{\circ}$ $55^{\prime} 48.2^{\prime \prime} \mathrm{N} 24^{\circ} 55^{\prime} 24.4^{\prime \prime}$ E), Rovinj (Croatia, 45 07' 03.7" N $13^{\circ} 36^{\prime} 59.4^{\prime \prime} \mathrm{E}$ ), and Lebanon (south of Tyre, $33^{\circ} 15^{\prime} 38.0^{\prime \prime} \mathrm{N}$ $35^{\circ} 12^{\prime} 31.2^{\prime \prime}$ E) at $0.3-3 \mathrm{~m}$ depth (Fig. 1). From Croatia, 30 specimens were collected from a rocky shore neighbouring a zone of macro algae. Marsalto Valley (30 specimens) represents a sheltered habitat with a solid rock ground, whereas Lentas and Dyticus Bay (two specimens each) have coarse gravel. Another 26 specimens from the Agia Pelagia area (very exposed, coarse gravel) and six specimens from Lebanon (artificial coastal protection boulders) were included (Table 2). The animals were anaesthetized with isotonic $\mathrm{MgCl}_{2}$ solution and then frozen at $-80{ }^{\circ} \mathrm{C}$ or preserved in $96 \%$ ethanol. Shells and tissues were separated and individualised. The shells were kept under dry conditions. DNA extractions (Tissue DNA Mini Kit PeqGOLD, VWR, Germany) from foot tissue and PCR reactions with COI primers LCO and HCO (Folmer et al. 1994) followed standard protocols. PCR reactions were cleaned up with the Cycle-Pure Kit (PeqGOLD, VWR, Germany). Sequencing was carried out on standard ABI Sequencers (VBC Biotech, Vienna, Austria). Shells, tissue, and DNA extractions are kept for further studies at the Department of Integrative Zoology, University of Vienna.

In addition to these new samples, the type material for the following species was examined: Phorcus articulatus (Lamarck 1822), Ph. richardi (Payraudeau 1826), Ph. turbinatus (von Born, 1778), Steromphala divaricata 


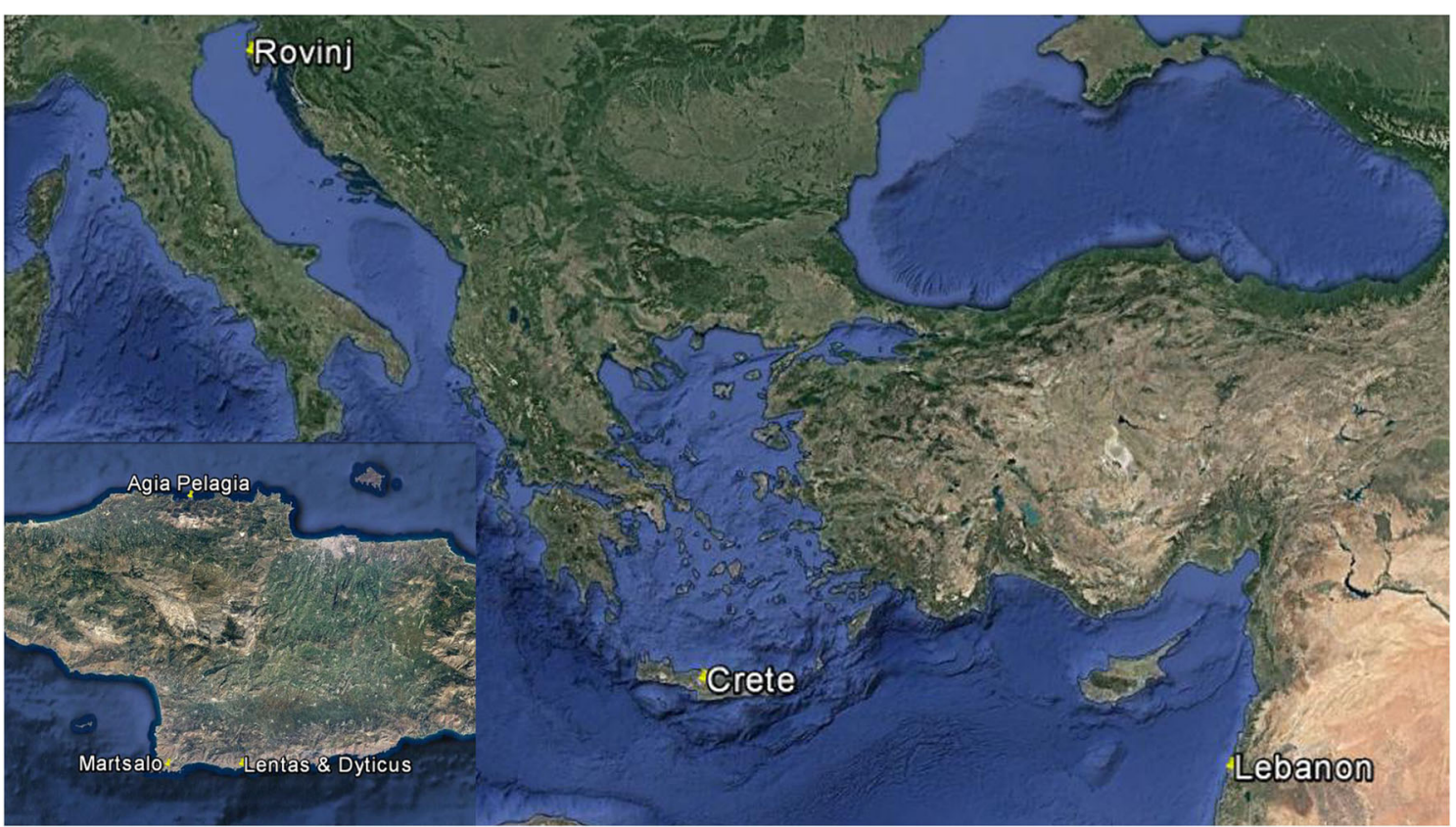

Fig. 1 Sampling sites for Mediterranean specimens of Steromphala and Phorcus species

(Linnaeus, 1758), St. umbilicaris (Linnaeus, 1758), St. varia (Linnaeus, 1758), St. adansonii (Payraudeau 1826), and St. cineraria (Linnaeus, 1758). However, as Steromphala cineraria does not occur in the Mediterranean (Gofas et al. 2011) and is clearly distinguished by its spiral ridges, it was excluded from the morphometric analyses.

The Linnean type material poses several problems. Although all species described by Linnaeus (1758) are represented in the collection of the Linnean Society London, it is not clear how many of them were used for the description or may have been added to the collection by later researchers and curators. Linneaus did not report numbers of specimens or individual type localities. Vague localities, such as "Mare Mediterraneo," provide no geographical clue for lectotype designations. We hope to demonstrate here that the chosen approach is suitable to link DNA-free types with extant specimens.

\section{Photography}

Shells were stabilised on a piece of clay and photographed in standardised lateral and ventral positions under identical optical settings with a Nikon SMZ 1500 stereomicroscope (Nikon Corp., Japan) (see Fig. 2). The shells in the Linnean Society London were photographed by one of the authors (SA). All other images of type material were provided by curators of type collections and taken under similar conditions. The lateral views are stacked in Adobe Photoshop (Adobe, Inc.) from up to 12 images at different focal depths. The lateral view shows the shell with the apex, the attachment point of the aperture on the previous whorl, and the umbilicus centre connected by an imaginary horizontal line. The ventral view is oriented in a way that the apex-umbilicus line is vertical.

\section{Morphometric analysis}

The images were landmarked in tps Dig2 2.16 (Rohlf 2010). The lateral view shows a set of 44 landmarks, including 29 semi-landmarks. The fixed landmarks in the lateral view (Fig. 2a) are the following: 1 = apex, 2 = leftmost point visible on the suture between last and second last whorl, 3 = attachment point of the aperture lip, 4 = rightmost point visible on the suture between ultimate and penultimate whorl, $5=$ crossing point of aperture
Table 2 Sampling sites and numbers of individuals collected

\begin{tabular}{|c|c|c|}
\hline Sampling site & \# of Individuals analysed & Coordinates \\
\hline Rovinj, Croatia & 30 & $45^{\circ} 07^{\prime} 03.7^{\prime \prime} \mathrm{N} 13^{\circ} 36^{\prime} 59.4^{\prime \prime} \mathrm{E}$ \\
\hline Agia Pelagia, Crete, Greece & 26 & $35^{\circ} 24^{\prime} 21.8^{\prime \prime} \mathrm{N} 24^{\circ} 50^{\prime} 07.7^{\prime \prime} \mathrm{E}$ \\
\hline Martsalo Valley, Crete, Greece & 30 & $34^{\circ} 55^{\prime} 48.0^{\prime \prime} \mathrm{N} 24^{\circ} 46^{\prime} 14.0^{\prime \prime} \mathrm{E}$ \\
\hline Lentas Bay, Crete, Greece & 2 & $34^{\circ} 55^{\prime} 48.2^{\prime \prime} \mathrm{N} 24^{\circ} 55^{\prime} 24.4^{\prime \prime} \mathrm{E}$ \\
\hline Dyticus Bay, Crete, Greece & 2 & $34^{\circ} 55^{\prime} 46.2^{\prime \prime} \mathrm{N} 24^{\circ} 54^{\prime} 45.9^{\prime \prime} \mathrm{E}$ \\
\hline Lebanon & 6 & $33^{\circ} 15^{\prime} 38.0^{\prime \prime} \mathrm{N} 35^{\circ} 12^{\prime} 31.2^{\prime \prime} \mathrm{E}$ \\
\hline
\end{tabular}




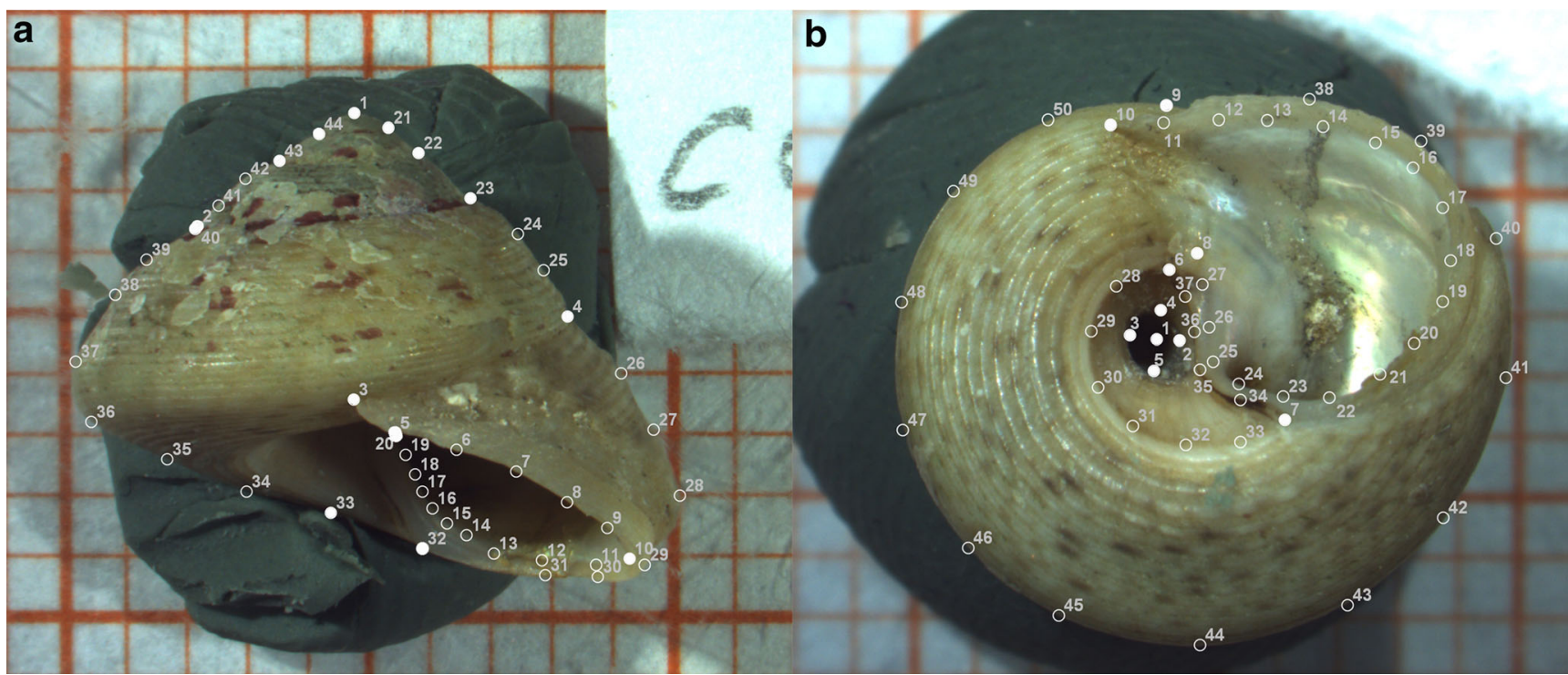

Fig. 2 Landmark templates for representative a lateral and $\mathbf{b}$ ventral standardised views. Filled circles show fixed landmarks. Empty circles show semilandmarks, processed as sliding landmarks

lip and columella, $10=$ leftmost point on the aperture lip, $20=$ last visible point of the columella, $21-23=$ leftmost points on the sutures, 32 = lowest point of the columellar fold, $33=$ centre of the umbilicus, $40=$ shoulder of the last whorl, and $43-$
$44=$ rightmost points of the sutures. The ventral view includes a set of 50 landmarks, 40 of which are semi-landmarks. The fixed landmarks in the lateral view (Fig. 2b) are defined as following: $1=$ centre of the umbilicus, $2=$ umbilicus edge closest to the

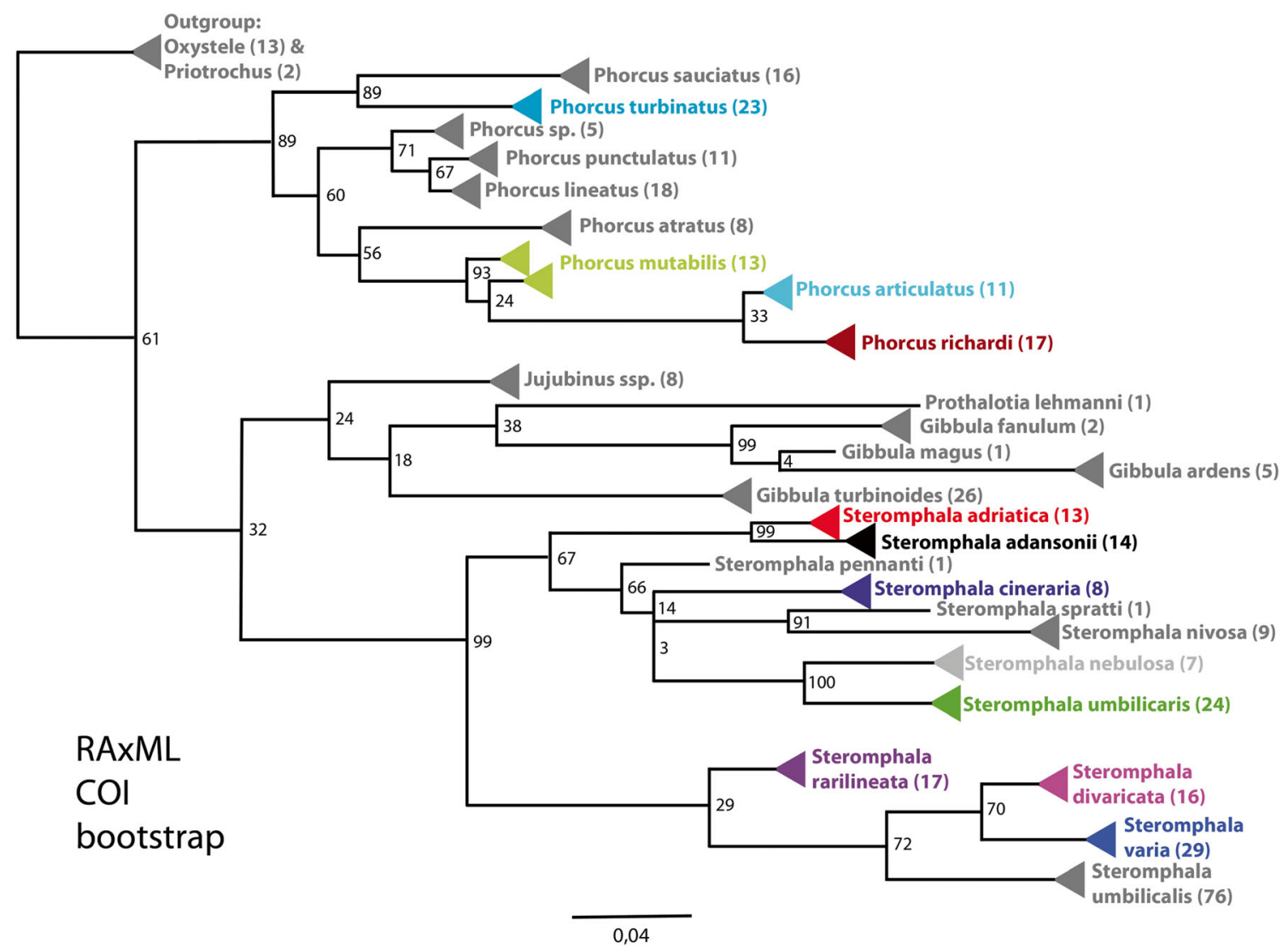

Fig. 3 RAxML based phylogenetic reconstruction (GTRGAMMA model and 1000 rapid bootstrap replicates). COI barcodes were used to identify species and reconstruct relationships between species and genera.
Number of individuals per species is in parentheses. Bootstrap values are given at nodes. Species evaluated in morphometric analysis are in corresponding colours (compare Table 4) 
Table 3 List of specimens used in the phylogenetic analyses

\begin{tabular}{|c|c|c|c|}
\hline Species & Voucher ID & Accession number $\mathrm{COI}$ & Reference \\
\hline Gibbula ardens (Salis, 1793) & $1075.4-5,10-11$ & $\begin{array}{l}\text { JQ839302-JQ839303, } \\
\text { JQ839309- JQ839310 }\end{array}$ & Barco et al. 2013 \\
\hline Gibbula ardens (Salis, 1793) & 1097.2 & JQ839306 & Barco et al. 2013 \\
\hline Gibbula fanulum (Gmelin, 1791) & 1088 & JQ839325 & Barco et al. 2013 \\
\hline Gibbula fanulum (Gmelin, 1791) & GFAN.KRC.1 & GQ232363 & Williams et al. 2010 \\
\hline Gibbula magus (Linnaeus, 1758) & GMAG.FAR.1 & GQ232364 & Williams et al. 2010 \\
\hline Gibbula turbinoides (Deshayes, 1835) & $1080.1-17,19-22,24-28$ & JQ839351-JQ839376 & Barco et al. 2013 \\
\hline Jujubinus exasperatus (Pennant, 1777) & JEXA.KRC.1 & GQ232368 & Williams et al. 2010 \\
\hline Jujubinus montagui (Wood, 1828) & KR084531 & KR084531 & Barco et al. 2016 \\
\hline Jujubinus montagui (Wood, 1828) & KR084831 & KR084831 & Barco et al. 2016 \\
\hline Jujubinus striatus (Linnaeus, 1758) & CSTR.CAL.1 & GQ232361 & Williams et al. 2010 \\
\hline Jujubinus striatus (Linnaeus, 1758) & $\operatorname{ech} 50$ & KJ183017 & Cowart et al. Unpublished \\
\hline Jujubinus striatus (Linnaeus, 1758) & KR084400 & KR084400 & Barco et al. 2016 \\
\hline Oxystele impervia (Menke, 1843) & South Africa & DQ061093 & Donald, Kennedy and Spencer 2005 \\
\hline Oxystele sinensis (Gmelin, 1791) & OsHB18 & JX303353 & Muteveri et al. Unpublished \\
\hline Oxystele sinensis (Gmelin, 1791) & OsHH03 & JX303372 & Muteveri et al. Unpublished \\
\hline Oxystele sinensis (Gmelin, 1791) & OsKH01 & JX303354 & Muteveri et al. Unpublished \\
\hline Oxystele sinensis (Gmelin, 1791) & OsPA23 & JX303371 & Muteveri et al. Unpublished \\
\hline Oxystele tabularis (Krauss, 1848) & South Africa & DQ061090 & Donald, Kennedey and Spencer 2005 \\
\hline Oxystele tigrina (Dillwyn, 1817) & OtHH01 & JX303448 & Muteveri et al. Unpublished \\
\hline Oxystele tigrina (Dillwyn, 1817) & OtKH24 & JX303442 & Muteveri et al. Unpublished \\
\hline Oxystele tigrina (Dillwyn, 1817) & OtPA09 & JX303447 & Muteveri et al. Unpublished \\
\hline Oxystele tigrina (Dillwyn, 1817) & OtPE18 & JX303443 & Muteveri et al. Unpublished \\
\hline Oxystele variegata (Anton, 1838) & $\mathrm{OvCA} 23$ & JX303471 & Muteveri et al. Unpublished \\
\hline Oxystele variegata (Anton, 1838) & OvHB01 & JX303472 & Muteveri et al. Unpublished \\
\hline Oxystele variegata (Anton, 1838) & OvKH17 & JX303485 & Muteveri et al. Unpublished \\
\hline Oxystele variegata (Anton, 1838) & OvPE05 & JX303486 & Muteveri et al. Unpublished \\
\hline Phorcus articulatus (Lamarck 1822) & 1 1Spain & JN686286 & Donald et al. 2012 \\
\hline Phorcus articulatus (Lamarck 1822) & 1a 1Tunisia & JN686283 & Donald et al. 2012 \\
\hline Phorcus articulatus (Lamarck 1822) & 1d 2Spain & JN686279 & Donald et al. 2012 \\
\hline Phorcus articulatus (Lamarck 1822) & 1 s 1Spain & JN686280 & Donald et al. 2012 \\
\hline Phorcus articulatus (Lamarck 1822) & 2 1Spain & JN686287 & Donald et al. 2012 \\
\hline Phorcus articulatus (Lamarck 1822) & 2a 2Tunisia & JN686284 & Donald et al. 2012 \\
\hline Phorcus articulatus (Lamarck 1822) & 3 Spain & JN686288 & Donald et al. 2012 \\
\hline Phorcus articulatus (Lamarck 1822) & 3a Tunisia & JN686285 & Donald et al. 2012 \\
\hline Phorcus articulatus (Lamarck 1822) & 5 1Spain & JN686289 & Donald et al. 2012 \\
\hline Phorcus articulatus (Lamarck 1822) & 6 2Tunisia & JN686290 & Donald et al. 2012 \\
\hline Phorcus articulatus (Lamarck 1822) & Lib13_Lebanon & KY364988 & this study \\
\hline Phorcus atratus (Wood, 1828) & 1 Spain & JN686291 & Donald et al. 2012 \\
\hline Phorcus atratus (Wood, 1828) & 1 Spain & JN686293 & Donald et al. 2012 \\
\hline Phorcus atratus (Wood, 1828) & 1 Spain & JN686295 & Donald et al. 2012 \\
\hline Phorcus atratus (Wood, 1828) & 1 Spain & JN686297 & Donald et al. 2012 \\
\hline Phorcus atratus (Wood, 1828) & 2 Spain & JN686294 & Donald et al. 2012 \\
\hline Phorcus atratus (Wood, 1828) & 2 Spain & JN686298 & Donald et al. 2012 \\
\hline Phorcus atratus (Wood, 1828) & 3 Spain & JN686292 & Donald et al. 2012 \\
\hline Phorcus atratus (Wood, 1828) & 3 Spain & JN686296 & Donald et al. 2012 \\
\hline Phorcus lineatus (da Costa, 1778) & 1 France & JN686331 & Donald et al. 2012 \\
\hline Phorcus lineatus (da Costa, 1778) & 1 Morocco & JN686325 & Donald et al. 2012 \\
\hline Phorcus lineatus (da Costa, 1778) & 1 Morocco & JN686333 & Donald et al. 2012 \\
\hline Phorcus lineatus (da Costa, 1778) & 1 Portugal & JN686317 & Donald et al. 2012 \\
\hline Phorcus lineatus (da Costa, 1778) & 1 Spain & JN686324 & Donald et al. 2012 \\
\hline Phorcus lineatus (da Costa, 1778) & 1 United Kingdom & JN686321 & Donald et al. 2012 \\
\hline Phorcus lineatus (da Costa, 1778) & 1 United Kingdom & JN686328 & Donald et al. 2012 \\
\hline Phorcus lineatus (da Costa, 1778) & 2 Morocco & JN686326 & Donald et al. 2012 \\
\hline Phorcus lineatus (da Costa, 1778) & 2 Portugal & JN686318 & Donald et al. 2012 \\
\hline Phorcus lineatus (da Costa, 1778) & 2 Portugal & JN686319 & Donald et al. 2012 \\
\hline Phorcus lineatus (da Costa, 1778) & 2 United Kingdom & JN686322 & Donald et al. 2012 \\
\hline Phorcus lineatus (da Costa, 1778) & 2 United Kingdom & JN686329 & Donald et al. 2012 \\
\hline Phorcus lineatus (da Costa, 1778) & 3 France & JN686332 & Donald et al. 2012 \\
\hline
\end{tabular}


Table 3 (continued)

\begin{tabular}{|c|c|c|c|}
\hline Species & Voucher ID & Accession number COI & Reference \\
\hline Phorcus lineatus (da Costa, 1778) & 3 Morocco & JN686327 & Donald et al. 2012 \\
\hline Phorcus lineatus (da Costa, 1778) & 3 Portugal & JN686320 & Donald et al. 2012 \\
\hline Phorcus lineatus (da Costa, 1778) & 3 United Kingdom & JN686323 & Donald et al. 2012 \\
\hline Phorcus lineatus (da Costa, 1778) & 3 United Kingdom & JN686330 & Donald et al. 2012 \\
\hline Phorcus lineatus (da Costa, 1778) & Figueras55 & JN241978 & Prado-Sanchez et al. Unpublished \\
\hline Phorcus mutabilis (Philippi, 1846) & 1 Italy & JN686364 & Donald et al. 2012 \\
\hline Phorcus mutabilis (Philippi, 1846) & 1 Turkey & JN686339 & Donald et al. 2012 \\
\hline Phorcus mutabilis (Philippi, 1846) & $1278.2,4,7$ & $\begin{array}{l}\text { JX887430, JX887455, } \\
\text { JX887446 }\end{array}$ & Barco et al. 2013 \\
\hline Phorcus mutabilis (Philippi, 1846) & 2 Italy & JN686365 & Donald et al. 2012 \\
\hline Phorcus mutabilis (Philippi, 1846) & C12_Croatia & KY364918 & this study \\
\hline Phorcus mutabilis (Philippi, 1846) & $\mathrm{C} 13^{-}$Croatia & KY364919 & this study \\
\hline Phorcus mutabilis (Philippi, 1846) & C14 Croatia & KY364920 & this study \\
\hline Phorcus mutabilis (Philippi, 1846) & C15_Croatia & KY364921 & this study \\
\hline Phorcus mutabilis (Philippi, 1846) & N02_NCrete & KY364934 & this study \\
\hline Phorcus mutabilis (Philippi, 1846) & N08_NCrete & KY364940 & this study \\
\hline Phorcus mutabilis (Philippi, 1846) & S24_SCrete & KY364978 & this study \\
\hline Phorcus punctulatus (Lamarck 1822) & 1 Senegal & JN686357 & Donald et al. 2012 \\
\hline Phorcus punctulatus (Lamarck 1822) & 10 Senegal & JN686350 & Donald et al. 2012 \\
\hline Phorcus punctulatus (Lamarck 1822) & 11 Senegal & JN686351 & Donald et al. 2012 \\
\hline Phorcus punctulatus (Lamarck 1822) & 12 Senegal & JN686352 & Donald et al. 2012 \\
\hline Phorcus punctulatus (Lamarck 1822) & 2 Senegal & JN686358 & Donald et al. 2012 \\
\hline Phorcus punctulatus (Lamarck 1822) & 3 Senegal & JN686359 & Donald et al. 2012 \\
\hline Phorcus punctulatus (Lamarck 1822) & 4 Senegal & JN686360 & Donald et al. 2012 \\
\hline Phorcus punctulatus (Lamarck 1822) & 5 Senegal & JN686353 & Donald et al. 2012 \\
\hline Phorcus punctulatus (Lamarck 1822) & 6 Senegal & JN686354 & Donald et al. 2012 \\
\hline Phorcus punctulatus (Lamarck 1822) & 8 Senegal & JN686355 & Donald et al. 2012 \\
\hline Phorcus punctulatus (Lamarck 1822) & 9 Senegal & JN686356 & Donald et al. 2012 \\
\hline Phorcus richardi (Payraudeau 1826) & 1 Croatia & JN686281 & Donald et al. 2012 \\
\hline Phorcus richardi (Payraudeau 1826) & 1 Spain, Almuñecar & JN686361 & Donald et al. 2012 \\
\hline Phorcus richardi (Payraudeau 1826) & 1 Spain, Cabo Raja & JN686362 & Donald et al. 2012 \\
\hline Phorcus richardi (Payraudeau 1826) & $1278.1,3,5,8$ & $\begin{array}{l}\text { JX887443, JX887433, } \\
\text { JX887457, JX887444 }\end{array}$ & Barco et al. 2013 \\
\hline Phorcus richardi (Payraudeau 1826) & 2 Croatia & $\mathrm{JN} 686282$ & Donald et al. 2012 \\
\hline Phorcus richardi (Payraudeau 1826) & 2 Spain & JN686363 & Donald et al. 2012 \\
\hline Phorcus richardi (Payraudeau 1826) & D01_SCrete & KY364929 & This study \\
\hline Phorcus richardi (Payraudeau 1826) & D02_SCrete & KY364930 & This study \\
\hline Phorcus richardi (Payraudeau 1826) & L01_SCrete & KY364931 & This study \\
\hline Phorcus richardi (Payraudeau 1826) & L02_SCrete & KY364932 & This study \\
\hline Phorcus richardi (Payraudeau 1826) & N01_NCrete & KY364933 & This study \\
\hline Phorcus richardi (Payraudeau 1826) & N03_NCrete & KY364935 & This study \\
\hline Phorcus richardi (Payraudeau 1826) & N04_NCrete & KY364936 & This study \\
\hline Phorcus richardi (Payraudeau 1826) & N05_NCrete & KY364937 & This study \\
\hline Phorcus richardi (Payraudeau 1826) & N06_NCrete & KY364938 & This study \\
\hline Phorcus richardi (Payraudeau 1826) & N07_NCrete & KY364939 & This study \\
\hline Phorcus richardi (Payraudeau 1826) & N09 NCrete & KY364941 & This study \\
\hline Phorcus richardi (Payraudeau 1826) & N10_NCrete & KY364942 & This study \\
\hline Phorcus richardi (Payraudeau 1826) & N11_NCrete & KY364943 & This study \\
\hline Phorcus richardi (Payraudeau 1826) & N12_NCrete & KY364944 & This study \\
\hline Phorcus richardi (Payraudeau 1826) & N13_NCrete & KY364945 & This study \\
\hline Phorcus richardi (Payraudeau 1826) & N14 NCrete & KY364946 & This study \\
\hline Phorcus richardi (Payraudeau 1826) & N15 NCrete & KY364947 & This study \\
\hline Phorcus richardi (Payraudeau 1826) & N16_NCrete & KY364948 & This study \\
\hline Phorcus richardi (Payraudeau 1826) & N17_NCrete & KY364949 & This study \\
\hline Phorcus richardi (Payraudeau 1826) & N18_NCrete & KY364950 & This study \\
\hline Phorcus richardi (Payraudeau 1826) & N19_NCrete & KY364951 & This study \\
\hline Phorcus richardi (Payraudeau 1826) & $\mathrm{N} 20 \_\mathrm{NCrete}$ & KY364952 & This study \\
\hline Phorcus richardi (Payraudeau 1826) & N21_NCrete & KY364953 & This study \\
\hline Phorcus richardi (Payraudeau 1826) & $\mathrm{N} 22 \_\mathrm{NCrete}$ & KY364954 & This study \\
\hline Phorcus richardi (Payraudeau 1826) & N23_NCrete & KY364955 & This study \\
\hline Phorcus richardi (Payraudeau 1826) & $\mathrm{N} 24$ NCrete & KY364956 & This study \\
\hline Phorcus richardi (Payraudeau 1826) & S02_SCrete & KY364958 & This study \\
\hline Phorcus richardi (Payraudeau 1826) & S03 SCrete & KY364959 & This study \\
\hline
\end{tabular}


Table 3 (continued)

\begin{tabular}{|c|c|c|c|}
\hline Species & Voucher ID & Accession number COI & Reference \\
\hline Phorcus richardi (Payraudeau 1826) & S04_SCrete & KY364960 & This study \\
\hline Phorcus richardi (Payraudeau 1826) & S06_SCrete & KY364962 & This study \\
\hline Phorcus richardi (Payraudeau 1826) & S07_SCrete & KY364963 & This study \\
\hline Phorcus richardi (Payraudeau 1826) & S08_SCrete & KY364964 & This study \\
\hline Phorcus richardi (Payraudeau 1826) & S09_SCrete & KY364965 & This study \\
\hline Phorcus richardi (Payraudeau 1826) & S13_SCrete & KY364968 & This study \\
\hline Phorcus richardi (Payraudeau 1826) & S14_SCrete & KY364969 & This study \\
\hline Phorcus richardi (Payraudeau 1826) & S15_SCrete & KY364970 & This study \\
\hline Phorcus richardi (Payraudeau 1826) & S16_SCrete & KY364971 & This study \\
\hline Phorcus richardi (Payraudeau 1826) & S19_SCrete & KY364973 & This study \\
\hline Phorcus richardi (Payraudeau 1826) & S20_SCrete & KY364974 & This study \\
\hline Phorcus richardi (Payraudeau 1826) & S21_SCrete & KY364975 & This study \\
\hline Phorcus richardi (Payraudeau 1826) & S27_SCrete & KY364980 & This study \\
\hline Phorcus richardi (Payraudeau 1826) & S29_SCrete & KY364982 & This study \\
\hline Phorcus richardi (Payraudeau 1826) & S30_SCrete & KY364983 & This study \\
\hline Phorcus sauciatus (Koch, 1845) & 1 Morocco & JN686307 & Donald et al. 2012 \\
\hline Phorcus sauciatus (Koch, 1845) & 1 Morocco & JN686312 & Donald et al. 2012 \\
\hline Phorcus sauciatus (Koch, 1845) & 1 Portugal & JN686300 & Donald et al. 2012 \\
\hline Phorcus sauciatus (Koch, 1845) & 1 Spain & JN686299 & Donald et al. 2012 \\
\hline Phorcus sauciatus (Koch, 1845) & 1 Spain & JN686305 & Donald et al. 2012 \\
\hline Phorcus sauciatus (Koch, 1845) & 1 Spain & JN686309 & Donald et al. 2012 \\
\hline Phorcus sauciatus (Koch, 1845) & 1 Spain & JN686314 & Donald et al. 2012 \\
\hline Phorcus sauciatus (Koch, 1845) & 2 Morocco & JN686308 & Donald et al. 2012 \\
\hline Phorcus sauciatus (Koch, 1845) & 2 Portugal & JN686306 & Donald et al. 2012 \\
\hline Phorcus sauciatus (Koch, 1845) & 2 Spain & JN686301 & Donald et al. 2012 \\
\hline Phorcus sauciatus (Koch, 1845) & 2 Spain & JN686303 & Donald et al. 2012 \\
\hline Phorcus sauciatus (Koch, 1845) & 2 Spain & JN686310 & Donald et al. 2012 \\
\hline Phorcus sauciatus (Koch, 1845) & 3 Morocco & JN686313 & Donald et al. 2012 \\
\hline Phorcus sauciatus (Koch, 1845) & 3 Spain & JN686302 & Donald et al. 2012 \\
\hline Phorcus sauciatus (Koch, 1845) & 3 Spain & JN686304 & Donald et al. 2012 \\
\hline Phorcus sauciatus (Koch, 1845) & 3 Spain & JN686311 & Donald et al. 2012 \\
\hline Phorcus sp & 1 Cape Verde & JN686334 & Donald et al. 2012 \\
\hline Phorcus sp & 1 Cape Verde & JN686337 & Donald et al. 2012 \\
\hline Phorcus sp & 2 Cape Verde & JN686338 & Donald et al. 2012 \\
\hline Phorcus sp & 3 Cape Verde & JN686336 & Donald et al. 2012 \\
\hline Phorcus sp & 2 Cape Verde & JN686335 & Donald et al. 2012 \\
\hline Phorcus turbinatus (Born, 1778) & 1 Croatia & JN686344 & Donald et al. 2012 \\
\hline Phorcus turbinatus (Born, 1778) & 1 Cyprus & JN686341 & Donald et al. 2012 \\
\hline Phorcus turbinatus (Born, 1778) & 1 Italy & JN686348 & Donald et al. 2012 \\
\hline Phorcus turbinatus (Born, 1778) & 1 Spain O.turbinatus1 3496 & JN686340 & Donald et al. 2012 \\
\hline Phorcus turbinatus (Born, 1778) & 1 Spain O.turbinatus2 & JN686343 & Donald et al. 2012 \\
\hline Phorcus turbinatus (Born, 1778) & 2 Spain & JN686346 & Donald et al. 2012 \\
\hline Phorcus turbinatus (Born, 1778) & 2 Turkey & JN686345 & Donald et al. 2012 \\
\hline Phorcus turbinatus (Born, 1778) & $283,993,123$ & GQ434018 & Donald et al. 2012 \\
\hline Phorcus turbinatus (Born, 1778) & 3 Cyprus & JN686342 & Donald et al. 2012 \\
\hline Phorcus turbinatus (Born, 1778) & 3 Spain & JN686347 & Donald et al. 2012 \\
\hline Phorcus turbinatus (Born, 1778) & 3Italy & JN686349 & Donald et al. 2012 \\
\hline Phorcus turbinatus (Born, 1778) & Lib02_Lebanon & KY364985 & this study \\
\hline Phorcus turbinatus (Born, 1778) & Lib03_Lebanon & KY364986 & this study \\
\hline Phorcus turbinatus (Born, 1778) & Lib04_Lebanon & KY364987 & this study \\
\hline Phorcus turbinatus (Born, 1778) & N26_Nōete & KY364989 & this study \\
\hline Phorcus turbinatus (Born, 1778) & N27_NCrete & KY364992 & this study \\
\hline Phorcus turbinatus (Born, 1778) & NHM̄UK20080408 & GQ434019 & Williams et al. 2010 \\
\hline Priotrochus kotschyi (Philippi 1849) & 1 Bahrain & JN686315 & Donald et al. 2012 \\
\hline Priotrochus kotschyi (Philippi 1849) & 3 Bahrain & JN686316 & Donald et al. 2012 \\
\hline Prothalotia lehmanni (Menke, 1843) & PLEH.DNS.1 & EU530123 & Williams, Karube and Ozawa 2008 \\
\hline Steromphala adansonii (Payraudeau 1826) & $1072.2-11$ & JQ839291-JQ839300 & Barco et al. 2013 \\
\hline Steromphala adansonii (Payraudeau 1826) & 1075.2 & JQ839290 & Barco et al. 2013 \\
\hline Steromphala adansonii (Payraudeau 1826) & $1284.3,4$ & JX887439, JX887458 & Barco et al. 2013 \\
\hline Steromphala adansonii (Payraudeau 1826) & C16_Croatia & KY364922 & This study \\
\hline Steromphala adriatica (Philippi, 1844) & S05_SCrete & KY364961 & This study \\
\hline
\end{tabular}


Table 3 (continued)

\begin{tabular}{|c|c|c|c|}
\hline Species & Voucher ID & Accession number COI & Reference \\
\hline Steromphala adriatica (Philippi, 1844) & S11_SCrete & KY364966 & This study \\
\hline Steromphala adriatica (Philippi, 1844) & $\mathrm{S} 12$ SCrete & KY364967 & This study \\
\hline Steromphala adriatica (Philippi, 1844) & S18_SCrete & KY364972 & This study \\
\hline Steromphala adriatica (Philippi, 1844) & S22_SCrete & KY364976 & This study \\
\hline Steromphala adriatica (Philippi, 1844) & S23_SCrete & KY364977 & This study \\
\hline Steromphala adriatica (Philippi, 1844) & S25_SCrete & KY364979 & This study \\
\hline Steromphala adriatica (Philippi, 1844) & S28_SCrete & KY364981 & This study \\
\hline Steromphala adriatica ${ }^{a}$ (Philippi, 1844) & 1260 & JX887463 & Barco et al. 2013 \\
\hline Steromphala adriatica ${ }^{\text {a }}$ (Philippi, 1844) & $1277.4,6$ & JX887465, JX887452 & Barco et al. 2013 \\
\hline Steromphala adriatica ${ }^{\mathrm{a}}$ (Philippi, 1844) & $1286.1,2,4,5$ & $\begin{array}{l}\text { JX887435, JX887453, } \\
\text { JX887431, KC417497 }\end{array}$ & Barco et al. 2013 \\
\hline Steromphala adriatica ${ }^{\mathrm{b}}$ (Philippi, 1844) & 146335560 & EF541179 & Samadi \& Steiner unpublished \\
\hline Steromphala cineraria (Linnaeus, 1758) & ech49 & KJ183016 & Cowart et al. Unpublished \\
\hline Steromphala cineraria (Linnaeus, 1758) & GCIN.WEM.1 & AM049339 & Williams and Ozawa 2006 \\
\hline Steromphala cineraria (Linnaeus, 1758) & MT09438 & KR084411 & Barco et al. 2016 \\
\hline Steromphala cineraria (Linnaeus, 1758) & МТ09439 & KR084951 & Barco et al. 2016 \\
\hline Steromphala cineraria (Linnaeus, 1758) & МТ09464 & KR084500 & Barco et al. 2016 \\
\hline Steromphala cineraria (Linnaeus, 1758) & МT09467 & KR084441 & Barco et al. 2016 \\
\hline Steromphala cineraria (Linnaeus, 1758) & MT09471 & KR084415 & Barco et al. 2016 \\
\hline Steromphala divaricata (Linnaeus, 1758) & $1076.1,3,4,7,11$ & $\begin{array}{l}\text { JQ839319, JQ839321- } \\
\text { JQ839324 }\end{array}$ & Barco et al. 2013 \\
\hline Steromphala divaricata (Linnaeus, 1758) & $1087.1-7$ & $\begin{array}{l}\text { JQ839313-JQ839318, } \\
\text { JQ839320 }\end{array}$ & Barco et al. 2013 \\
\hline Steromphala divaricata (Linnaeus, 1758) & BAU1253 Italy & KC417500 & Barco et al. 2013 \\
\hline Steromphala divaricata (Linnaeus, 1758) & C17 Croatia & KY364923 & This study \\
\hline Steromphala divaricata (Linnaeus, 1758) & C20_Croatia & KY364924 & This study \\
\hline Steromphala divaricata (Linnaeus, 1758) & C21_Croatia & KY364925 & This study \\
\hline Steromphala divaricata (Linnaeus, 1758) & C22_Croatia & KY364926 & This study \\
\hline Steromphala divaricata (Linnaeus, 1758) & C24_Croatia & KY364928 & This study \\
\hline Steromphala nebulosa (Philippi, 1848) & $108 \overline{6} .5,71098.2$ & $\begin{array}{l}\text { JQ8393228, JQ8393230, } \\
\text { JQ839327 }\end{array}$ & Barco et al. 2013 \\
\hline Steromphala nebulosa (Philippi, 1848) & C35 Croatia & KY364991 & this study \\
\hline Steromphala nivosa (Adams, 1853) & $101 \overline{8} .1-3,1078.1-6$ & JQ839332-JQ839340 & Barco et al. 2013 \\
\hline Steromphala pennanti (Pennanti, 1846) & GPEN.RSF.1 & GQ232365 & Williams et al. 2010 \\
\hline Steromphala rarilineata (Michaud, 1829) & 1076.8 & JQ839344 & Barco et al. 2013 \\
\hline Steromphala rarilineata (Michaud, 1829) & $1079.1-8$ & $\begin{array}{l}\text { JQ839341-JQ839343, } \\
\text { JQ839345 -JQ839349 }\end{array}$ & Barco et al. 2013 \\
\hline Steromphala rarilineata (Michaud, 1829) & $1279.1-3,5,6$ & $\begin{array}{l}\text { JX887451, JX887466, } \\
\text { JX887467, JX887471 }\end{array}$ & Barco et al. 2013 \\
\hline Steromphala rarilineata (Michaud, 1829) & 1287.6 & $\mathrm{JX} 887450$ & Barco et al. 2013 \\
\hline Steromphala rarilineata (Michaud, 1829) & C23_Croatia & KY364927 & this study \\
\hline Steromphala rarilineata (Michaud, 1829) & GRĀR.KRC.1 & GQ232366 & Williams et al. 2010 \\
\hline Steromphala rarilineata (Michaud, 1829) & S01_SCrete & KY364957 & this study \\
\hline Steromphala spratti (Forbes, 1844) & $110 \overline{8}$ & JQ839350 & Barco et al. 2013 \\
\hline Steromphala umbilicalis (da Costa, 1778) & $1 \mathrm{UK}$ & JN686273 & Donald et al. 2012 \\
\hline Steromphala umbilicalis (da Costa, 1778) & $1 \mathrm{~A} 10$ & КР064694 & Muñoz-Colmenero et al. 2015 \\
\hline Steromphala umbilicalis (da Costa, 1778) & $1 \mathrm{~A} 11$ & KP064695 & Muñoz-Colmenero et al. 2015 \\
\hline Steromphala umbilicalis (da Costa, 1778) & $1 \mathrm{~A} 12$ & KP064696 & Muñoz-Colmenero et al. 2015 \\
\hline Steromphala umbilicalis (da Costa, 1778) & $1 \mathrm{~A} 14$ & KP064697 & Muñoz-Colmenero et al. 2015 \\
\hline Steromphala umbilicalis (da Costa, 1778) & $1 \mathrm{~A} 15$ & KP064698 & Muñoz-Colmenero et al. 2015 \\
\hline Steromphala umbilicalis (da Costa, 1778) & $1 \mathrm{~A} 18$ & КР064699 & Muñoz-Colmenero et al. 2015 \\
\hline Steromphala umbilicalis (da Costa, 1778) & $1 \mathrm{~A} 19$ & KP064700 & Muñoz-Colmenero et al. 2015 \\
\hline Steromphala umbilicalis (da Costa, 1778) & $1 \mathrm{~A} 22$ & KP064701 & Muñoz-Colmenero et al. 2015 \\
\hline Steromphala umbilicalis (da Costa, 1778) & $1 \mathrm{~A} 23$ & KP064702 & Muñoz-Colmenero et al. 2015 \\
\hline Steromphala umbilicalis (da Costa, 1778) & $1 \mathrm{~A} 29$ & KP064703 & Muñoz-Colmenero et al. 2015 \\
\hline Steromphala umbilicalis (da Costa, 1778) & $1 \mathrm{~A} 5$ & KP064704 & Muñoz-Colmenero et al. 2015 \\
\hline Steromphala umbilicalis (da Costa, 1778) & $1 \mathrm{~A} 7$ & KP064705 & Muñoz-Colmenero et al. 2015 \\
\hline Steromphala umbilicalis (da Costa, 1778) & $1 \mathrm{G} 7$ & KP064706 & Muñoz-Colmenero et al. 2015 \\
\hline Steromphala umbilicalis (da Costa, 1778) & $1 \mathrm{G} 8$ & KP064707 & Muñoz-Colmenero et al. 2015 \\
\hline Steromphala umbilicalis (da Costa, 1778) & 2 Portugal & JN686275 & Donald et al. 2012 \\
\hline Steromphala umbilicalis (da Costa, 1778) & 2 Spain & JN686277 & Donald et al. 2012 \\
\hline Steromphala umbilicalis (da Costa, 1778) & $2 \mathrm{UK}$ & JN686274 & Donald et al. 2012 \\
\hline
\end{tabular}


Table 3 (continued)

\begin{tabular}{|c|c|c|c|}
\hline Species & Voucher ID & Accession number $\mathrm{COI}$ & Reference \\
\hline Steromphala umbilicalis (da Costa, 1778) & 3 Portugal & JN686276 & Donald et al. 2012 \\
\hline Steromphala umbilicalis (da Costa, 1778) & 3 Spain & JN686278 & Donald et al. 2012 \\
\hline Steromphala umbilicalis (da Costa, 1778) & 3A19 & KP064708 & Muñoz-Colmenero et al. 2015 \\
\hline Steromphala umbilicalis (da Costa, 1778) & $3 \mathrm{~A} 52$ & KP064709 & Muñoz-Colmenero et al. 2015 \\
\hline Steromphala umbilicalis (da Costa, 1778) & $5 \mathrm{~A} 12$ & KP064710 & Muñoz-Colmenero et al. 2015 \\
\hline Steromphala umbilicalis (da Costa, 1778) & $5 \mathrm{~A} 5$ & KP064711 & Muñoz-Colmenero et al. 2015 \\
\hline Steromphala umbilicalis (da Costa, 1778) & $5 \mathrm{~A} 8$ & KP064712 & Muñoz-Colmenero et al. 2015 \\
\hline Steromphala umbilicalis (da Costa, 1778) & $5 \mathrm{~A} 9$ & KP064713 & Muñoz-Colmenero et al. 2015 \\
\hline Steromphala umbilicalis (da Costa, 1778) & $5 \mathrm{G} 106$ & KР064714 & Muñoz-Colmenero et al. 2015 \\
\hline Steromphala umbilicalis (da Costa, 1778) & $5 \mathrm{G} 107$ & КР064715 & Muñoz-Colmenero et al. 2015 \\
\hline Steromphala umbilicalis (da Costa, 1778) & $5 \mathrm{G} 110$ & KP064716 & Muñoz-Colmenero et al. 2015 \\
\hline Steromphala umbilicalis (da Costa, 1778) & $5 \mathrm{G} 111$ & КР064717 & Muñoz-Colmenero et al. 2015 \\
\hline Steromphala umbilicalis (da Costa, 1778) & $5 \mathrm{G} 116$ & KP064718 & Muñoz-Colmenero et al. 2015 \\
\hline Steromphala umbilicalis (da Costa, 1778) & $5 \mathrm{G} 149$ & KP064719 & Muñoz-Colmenero et al. 2015 \\
\hline Steromphala umbilicalis (da Costa, 1778) & $5 \mathrm{G} 150$ & KP064720 & Muñoz-Colmenero et al. 2015 \\
\hline Steromphala umbilicalis (da Costa, 1778) & $5 \mathrm{G} 156$ & KP064721 & Muñoz-Colmenero et al. 2015 \\
\hline Steromphala umbilicalis (da Costa, 1778) & $5 \mathrm{G} 166$ & KP064722 & Muñoz-Colmenero et al. 2015 \\
\hline Steromphala umbilicalis (da Costa, 1778) & $5 \mathrm{G} 40$ & КР064723 & Muñoz-Colmenero et al. 2015 \\
\hline Steromphala umbilicalis (da Costa, 1778) & $5 \mathrm{G} 53$ & KP064724 & Muñoz-Colmenero et al. 2015 \\
\hline Steromphala umbilicalis (da Costa, 1778) & $5 \mathrm{G} 56$ & KP064725 & Muñoz-Colmenero et al. 2015 \\
\hline Steromphala umbilicalis (da Costa, 1778) & $5 \mathrm{G} 65$ & KP064726 & Muñoz-Colmenero et al. 2015 \\
\hline Steromphala umbilicalis (da Costa, 1778) & $5 \mathrm{G} 69$ & KP064727 & Muñoz-Colmenero et al. 2015 \\
\hline Steromphala umbilicalis (da Costa, 1778) & $5 \mathrm{G} 77$ & KP064728 & Muñoz-Colmenero et al. 2015 \\
\hline Steromphala umbilicalis (da Costa, 1778) & $5 \mathrm{G} 95$ & KP064729 & Muñoz-Colmenero et al. 2015 \\
\hline Steromphala umbilicalis (da Costa, 1778) & $5 \mathrm{G} 97$ & KP064730 & Muñoz-Colmenero et al. 2015 \\
\hline Steromphala umbilicalis (da Costa, 1778) & $6 \mathrm{~A} 10$ & KP064731 & Muñoz-Colmenero et al. 2015 \\
\hline Steromphala umbilicalis (da Costa, 1778) & $6 \mathrm{~A} 14$ & KP064732 & Muñoz-Colmenero et al. 2015 \\
\hline Steromphala umbilicalis (da Costa, 1778) & $6 \mathrm{~A} 4$ & KP064733 & Muñoz-Colmenero et al. 2015 \\
\hline Steromphala umbilicalis (da Costa, 1778) & $6 \mathrm{G} 11$ & KP064734 & Muñoz-Colmenero et al. 2015 \\
\hline Steromphala umbilicalis (da Costa, 1778) & $6 \mathrm{G} 8$ & KP064735 & Muñoz-Colmenero et al. 2015 \\
\hline Steromphala umbilicalis (da Costa, 1778) & $7 \mathrm{G} 11$ & KP064736 & Muñoz-Colmenero et al. 2015 \\
\hline Steromphala umbilicalis (da Costa, 1778) & $7 \mathrm{G} 12$ & КР064737 & Muñoz-Colmenero et al. 2015 \\
\hline Steromphala umbilicalis (da Costa, 1778) & $7 \mathrm{G} 19$ & KP064738 & Muñoz-Colmenero et al. 2015 \\
\hline Steromphala umbilicalis (da Costa, 1778) & $8 \mathrm{~A} 1$ & KP064739 & Muñoz-Colmenero et al. 2015 \\
\hline Steromphala umbilicalis (da Costa, 1778) & $8 \mathrm{~A} 12$ & KP064740 & Muñoz-Colmenero et al. 2015 \\
\hline Steromphala umbilicalis (da Costa, 1778) & $8 \mathrm{~A} 15$ & KP064741 & Muñoz-Colmenero et al. 2015 \\
\hline Steromphala umbilicalis (da Costa, 1778) & $8 \mathrm{~A} 17$ & KP064742 & Muñoz-Colmenero et al. 2015 \\
\hline Steromphala umbilicalis (da Costa, 1778) & $8 \mathrm{~A} 20$ & KP064743 & Muñoz-Colmenero et al. 2015 \\
\hline Steromphala umbilicalis (da Costa, 1778) & $8 \mathrm{~A} 24$ & KP064744 & Muñoz-Colmenero et al. 2015 \\
\hline Steromphala umbilicalis (da Costa, 1778) & $8 \mathrm{~A} 3$ & KP064745 & Muñoz-Colmenero et al. 2015 \\
\hline Steromphala umbilicalis (da Costa, 1778) & $8 \mathrm{~A} 30$ & KP064746 & Muñoz-Colmenero et al. 2015 \\
\hline Steromphala umbilicalis (da Costa, 1778) & $8 \mathrm{G} 1$ & KP064747 & Muñoz-Colmenero et al. 2015 \\
\hline Steromphala umbilicalis (da Costa, 1778) & $8 \mathrm{G} 13$ & KP064748 & Muñoz-Colmenero et al. 2015 \\
\hline Steromphala umbilicalis (da Costa, 1778) & $8 \mathrm{G} 16$ & КР064749 & Muñoz-Colmenero et al. 2015 \\
\hline Steromphala umbilicalis (da Costa, 1778) & $8 \mathrm{G} 17$ & KP064750 & Muñoz-Colmenero et al. 2015 \\
\hline Steromphala umbilicalis (da Costa, 1778) & $8 \mathrm{G} 20$ & KP064751 & Muñoz-Colmenero et al. 2015 \\
\hline Steromphala umbilicalis (da Costa, 1778) & $8 \mathrm{G} 23$ & KP064752 & Muñoz-Colmenero et al. 2015 \\
\hline Steromphala umbilicalis (da Costa, 1778) & $8 \mathrm{G} 26$ & KP064753 & Muñoz-Colmenero et al. 2015 \\
\hline Steromphala umbilicalis (da Costa, 1778) & $8 \mathrm{G} 36$ & KP064754 & Muñoz-Colmenero et al. 2015 \\
\hline Steromphala umbilicalis (da Costa, 1778) & $8 \mathrm{G} 5$ & KP064755 & Muñoz-Colmenero et al. 2015 \\
\hline Steromphala umbilicalis (da Costa, 1778) & $8 \mathrm{G} 8$ & KP064756 & Muñoz-Colmenero et al. 2015 \\
\hline Steromphala umbilicalis (da Costa, 1778) & GN7 & KJ818224 & Muñoz-Colmenero et al. 2015 \\
\hline Steromphala umbilicalis (da Costa, 1778) & GUMB.WEM.1 & GQ232367 & Williams et al. 2010 \\
\hline Steromphala umbilicalis (da Costa, 1778) & SPedro13, 2, 4, 5, 6 & $\begin{array}{l}\text { JN241975, JN241973, } \\
\text { JN241976, JN241977, } \\
\text { JN241974 }\end{array}$ & Prado-Sanchez et al. Unpublished \\
\hline Steromphala umbilicaris (Linnaeus, 1758) & $1083.2-8$ & JQ839383-JQ839389 & Barco et al. 2013 \\
\hline Steromphala umbilicaris (Linnaeus, 1758) & $1084.1-5$ & JQ839378-JQ839381, & Barco et al. 2013 \\
\hline
\end{tabular}


Table 3 (continued)

\begin{tabular}{|c|c|c|c|}
\hline Species & Voucher ID & Accession number COI & Reference \\
\hline Steromphala umbilicaris (Linnaeus, 1758) & $1086.2,3$ & JQ839382, JQ839377 & Barco et al. 2013 \\
\hline Steromphala umbilicaris (Linnaeus, 1758) & $1275.1,2,6,8-11$ & $\begin{array}{l}\text { JX887442, JX887447, } \\
\text { JX887461, JX887468- } \\
\text { JX887470, JX887472 }\end{array}$ & Barco et al. 2013 \\
\hline Steromphala umbilicaris (Linnaeus, 1758) & 1286.6 & JX887428 & Barco et al. 2013 \\
\hline Steromphala umbilicaris (Linnaeus, 1758) & C26_Croatia & KY364993 & this study \\
\hline Steromphala umbilicaris (Linnaeus, 1758) & $\mathrm{C} 27$ Croatia & KY364990 & this study \\
\hline Steromphala umbilicaris (Linnaeus, 1758) & C34_Croatia & KY364994 & this study \\
\hline Steromphala varia (Linnaeus, 1758) & $125 \overline{7}$ & KC417496 & Barco et al. 2013 \\
\hline Steromphala varia (Linnaeus, 1758) & $1081.1,3$ & JQ839395, JQ839391 & Barco et al. 2013 \\
\hline Steromphala varia (Linnaeus, 1758) & $1082.3-5$ & JQ839392- JQ839394 & Barco et al. 2013 \\
\hline Steromphala varia (Linnaeus, 1758 ) & $1262.1-2$ & JX887438, JX887429 & Barco et al. 2013 \\
\hline Steromphala varia (Linnaeus, 1758) & $1276.1-7,9,11-13,16-18$ & $\begin{array}{c}\text { JX887427, JX887432, } \\
\text { JX887434, JX887437, } \\
\text { JX887440, JX887441, } \\
\text { JX887448, JX887449, } \\
\text { JX887454, JX887456, } \\
\text { JX887459, JX887460, } \\
\text { JX887462, JX887464 }\end{array}$ & Barco et al. 2013 \\
\hline Steromphala varia (Linnaeus, 1758) & C01_Croatia & KY364908 & This study \\
\hline Steromphala varia (Linnaeus, 1758) & C02_Croatia & KY364909 & This study \\
\hline Steromphala varia (Linnaeus, 1758) & C03_Croatia & KY364910 & This study \\
\hline Steromphala varia (Linnaeus, 1758) & $\mathrm{C} 04^{-}$Croatia & KY364911 & This study \\
\hline Steromphala varia (Linnaeus, 1758) & C05_Croatia & KY364912 & This study \\
\hline Steromphala varia (Linnaeus, 1758) & C06_Croatia & KY364913 & This study \\
\hline Steromphala varia (Linnaeus, 1758 ) & C07_Croatia & KY364914 & This study \\
\hline Steromphala varia (Linnaeus, 1758) & C08_Croatia & KY364915 & This study \\
\hline Steromphala varia (Linnaeus, 1758) & C09_Croatia & KY364916 & This study \\
\hline Steromphala varia (Linnaeus, 1758) & C10_Croatia & KY364917 & This study \\
\hline Steromphala varia (Linnaeus, 1758) & Lib01_Lebanon & KY364984 & This study \\
\hline
\end{tabular}

${ }^{\mathrm{a}}$ Genbank entry as Gibbula adansonii

${ }^{\mathrm{b}}$ Genbank entry as Gibbula varia

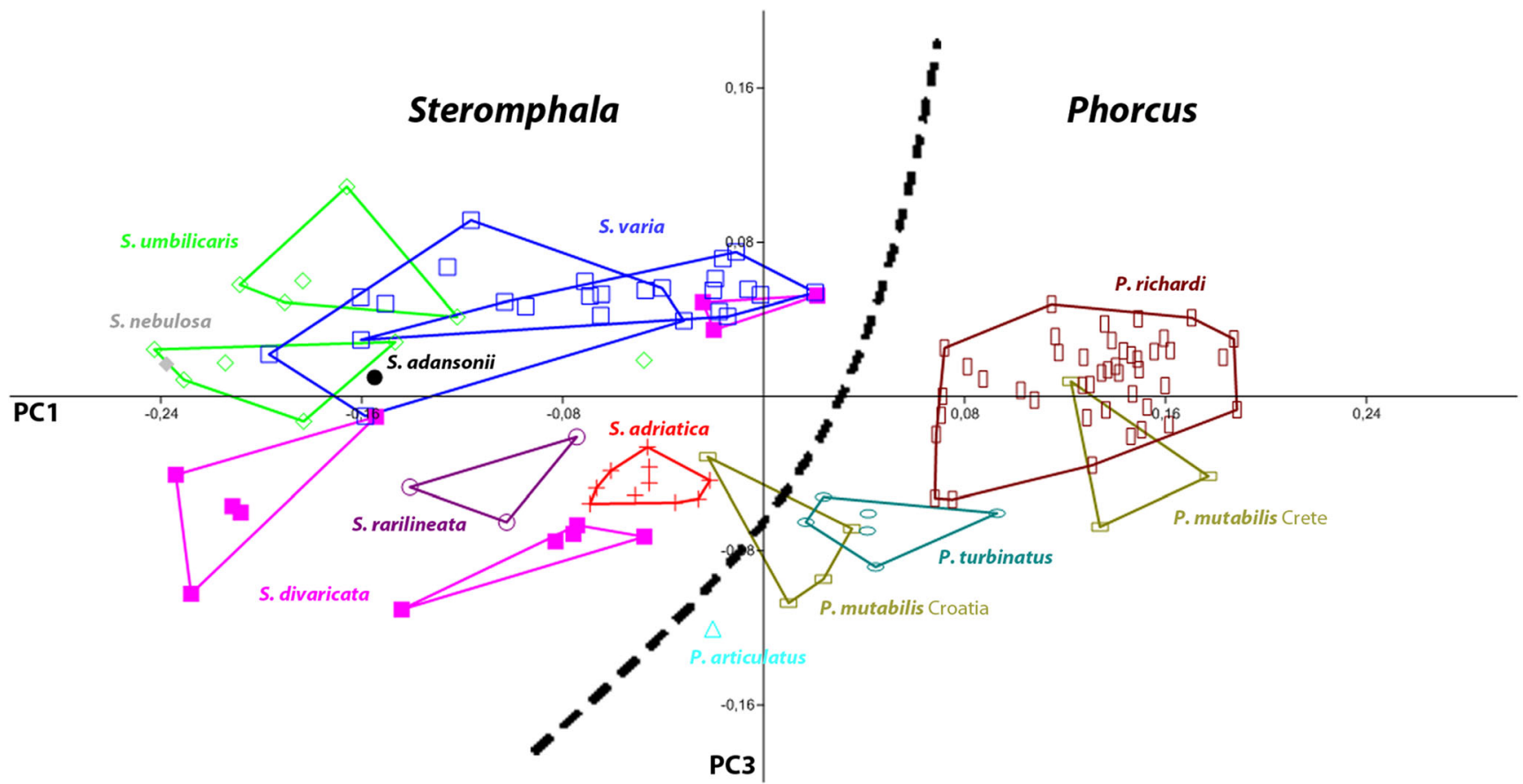

Fig. 4 Plot of first and third principal component of combined lateral and ventral landmark data from Phorcus and Steromphala individuals. A morphological separation of the two genera becomes apparent 


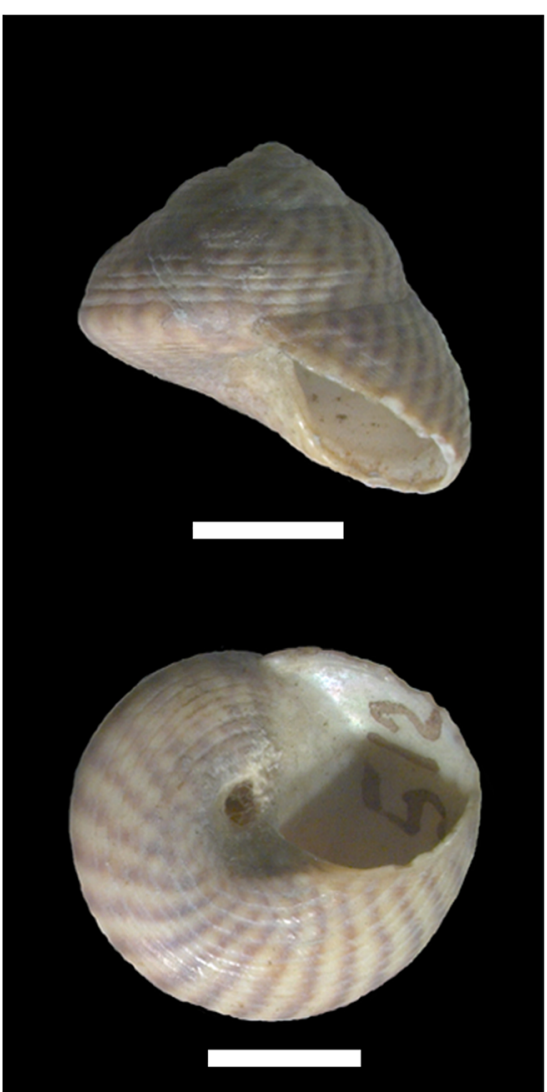

Fig. 5 Lectotype of Steromphala cineraria (Linnaeus, 1758). Located at LSL (LSL.502). Scale bar $5 \mathrm{~mm}$

columella, 3 = umbilicus edge facing LM2, 4 = umbilicus edge closest to basis of columella end, 5 = umbilicus edge facing LM4, $6=$ basis of the columella at umbilicus side, $7=$ uppermost point of umbilicus atrium, $8=$ basis of columella at aperture side, $9=$ crossing point of aperture lip and shell edge, and $10=$ attachment point of the aperture lip. After an initial round of setting landmarks on all pictures, all authors checked the complete data set for missed landmarks and correct positioning.

Semi-landmarks were defined as sliding landmarks in tps util (Rohlf 2009). The generalised Procrustes fit was carried out in tps relw (Rohlf 2005). Aligned landmark sets were checked for outliers in an XY-plot. The ventral and lateral coordinate sets were concatenated for a combined analysis. The aligned specimen coordinates were processed via a principal components analysis in PAST 2.12 (Hammer et al., 2001). First three principal components were chosen based on scree plots and separation in scatter plots.

COI sequences were checked for contamination by BLAST searches and then aligned with available sequences of this group in ClustalX. Sequences of Priotrochus and Oxystele served as outgroups (Table 3). Maximum Likelihood analyses were made in raxmlGUI (Silvestro and Michalak 2011) using the GTRGAMMA model with ML + 1000 rapid bootstrap replicates (See Online Resources for the reduced alignment in relaxed PHYLIP format and the ML-tree in pdf format).

\section{Results}

In the phylogenetic reconstruction based on the COI gene, the genera Jujubinus and Prothalotia dissolve the monophyly of Gibbula. The clade of the Gibbula type species, G. magus, includes the species $G$. ardens and $G$. fanulum. The type species Steromphala cineraria, St. adriatica, St. adansonii, St. pennanti,

Table 4 Species names assigned to specimen shells according to phylogenetic reconstruction based on COI sequences

\begin{tabular}{llll}
\hline Specimen ID & Species & Colour & Collection site \\
\hline Lib13 & Phorcus articulatus & Turquoise & Lebanon \\
C12-C15, N02, N08, S24 & Phorcus mutabilis & Olive & Croatia, Crete north, Crete south \\
D01-D02, L01-L02, N01, N03-N07, N09-N24, & Phorcus richardi & Maroon & Crete north, Crete south \\
$\quad$ S02-S04, S06-S09, S13-S16, S19-S21, S27, & & & Crete north, Lebanon \\
S29-S30 & & Petrol & Croatia \\
C16 & Phorcus turbinatus & Black & Crete south \\
S05, S11-S12, S18, S22-S23, S25, S26*, S28 & Steromphala adansonii & Red & Croatia, \\
Linne26-Linne35 & Steromphala adriatica & Dark blue & Croatia \\
C17, C20-C22, C24, Linne36-Linne43 & Steromphala cineraria & Pink & Croatia, Crete south \\
C35 & Steromphala divaricata & Grey & Croatia \\
C23, S01, S10* & Steromphala nebulosa & Violet & Croatia, Lebanon,- \\
C18*, C26-C27, C33*, C34, Linne20-Linne25 & Steromphala rarilineata & Green & Blue \\
C01-C10, Lib01, Linne06-Linne19 & Steromphala varia & & \\
\hline
\end{tabular}

Specimens marked with * were identified by shell characters only. Colour codes assigned to species as used in morphometric analyses. Specimens were coded for collection sites with running numbers: C Croatia, N Crete north, S Crete south, Lib Lebanon, D Crete south Dyticus, L Crete south Lentas, Linne type collection LSL 
Table 5 Variance explained by the first three axes of principal component analyses for combined lateral and ventral landmark sets of Steromphala, Phorcus, and both

\begin{tabular}{llll}
\hline & PC1 & PC2 & PC3 \\
\hline Steromphala + Phorcus & $49.37 \%$ & $13.64 \%$ & $7.57 \%$ \\
Steromphala & $32.04 \%$ & $21.86 \%$ & $13.45 \%$ \\
Phorcus & $30.17 \%$ & $21.07 \%$ & $7.96 \%$ \\
\hline
\end{tabular}

St. spratti, St. nivosa, St. nebulosa, St. umbilicaris, St. rarilineata, St. umbilicalis, St. divaricata, and St. varia are nested in a second clade, Steromphala Gray, 1847. The genus Phorcus is monophyletic. The genus clades Phorcus, Gibbula, and Steromphala show robust bootstrap support (Fig. 3).

The individuals comprising this study were assigned to 11 nominal species (Fig. 3). These species allocations (Table 4) were used in the geometric morphometric analyses.

One specimen is placed in Steromphala adansonii. Eight individuals are classified as St. adriatica. Five specimens clustered within St. divaricata and two into St. rarilineata. Eleven individuals were barcoded as St. varia. Steromphala umbilicaris was assigned to three specimens. One individual was identified as St. nebulosa. Additionally, four shells were morphologically identified as St. adriatica, St. rarilineata, and St. umbilicaris without molecular data (compare Table 4). A total of 35 specimens were assigned to the genus Steromphala.

The remaining individuals split into seven Phorcus mutabilis and 43 Ph. richardi. Phorcus articulatus is represented by a single individual from Lebanon. Six specimens were identified as $P h$. turbinatus, including one morphologically identified individual. The genus Phorcus was assigned to 57 individuals in this study.

The Linnean type specimens used for morphometric analyses were treated as separate species groups.

The combined data of lateral and ventral views of all taxa yield a first principal component axis (PC) explaining $49.37 \%$ of the variance in shell shape. The second and third PC explain 13.64 and $7.57 \%$, respectively (Table 5). In total, the first three PCs cover $70.58 \%$ of variance. Subsequent PCs provided little further information and no separation of taxa and were, thus, disregarded. In this analysis, a morphological separation of Steromphala and Phorcus is achieved (Fig. 4). To improve the resolution of the species within these two genera, separate principal component analyses were carried out for the Phorcus and Steromphala data sets. The strictly Atlantic St. cineraria was excluded (Fig. 5).

The principal component analysis of the combined lateral and ventral landmark set of the genus Steromphala shows the first PC with $32.04 \%$, the second PC with $21.86 \%$, and the third PC with $13.45 \%$ variance (Table 5), covering $67.35 \%$ of total shell shape variance.

The Steromphala analysis separates Steromphala umbilicaris from the other species. The type material and the recent specimens cluster together. The single specimen of St. nebulosa nests within this cluster (Fig. 6a). The single specimen of St. adansonii always separates from all other species (Fig. 7a). Steromphala adriatica, St. divaricata, and St. varia cluster with some overlap. S. adriatica is separated on the third PC (Fig. 8). Likewise, St. rarilineata nests within St. divaricata on the first two PCs but is set apart on the third PC (Fig. 9a). Three individuals of the type material of St. divaricata were re-identified as St. varia according to the characteristic umbilical atrium and cluster with the other St. varia specimens (Figs. 10a and 11a). All Steromphala species can be recovered as separate groups in the first three principal components, with the exception of St. divaricata, which overlaps slightly with St. varia in the first two principal components and with St. adriatica in the third principal component (Figs. 6, 7, 8, 9, 10 and 11).

In the principal component analysis for the genus Phorcus, the first PC explains $30.17 \%$, the second PC explains $21.07 \%$, and the third PC explains $7.96 \%$ of variance (Table 5), covering $59.2 \%$ of total in shell shape variance.

The species of the genus Phorcus are mostly recovered as separate clusters in the first three principal components. Phorcus richardi is the most abundant species in the sample and shows some variance in shell shape but can be recovered as a single species (Fig. 12). Only a single specimen from Crete, genetically unambiguously assigned to Phorcus mutabilis, shows a shell shape more similar to Ph. richardi (Fig. 13). Phorcus mutabilis from Crete is inseparable from $P h$. richardi on the first and second principal component axes but shows some separation on the third. Phorcus mutabilis from Croatia forms a distinct cluster without overlap with the Crete group (Fig. 13). The cluster of Phorcus turbinatus is well separated from all other species in the first two principal components (Fig. 14). The single specimen of Ph. articulatus is separated from all other species (Fig. 15).

\section{Discussion}

\section{Methods for species delineation}

Geometric morphometrics offer a tool for species delineation by objective, mathematical characterisation of shell shape. The ventral views show important characteristics of the umbilicus and its atrium where the lateral view captures the shape of the shell whorls. The combination of both views into a single data set offers the possibility to combine characteristics that cannot be captured in a single photo.

In general, the applicability of geometric morphometrics to species delineation in trochoid gastropods depends mainly on the standardising of views. Small rotations in the lateral view and tilting in ventral orientation bias results. Lateral and ventral views with 44 and 50 landmarks, respectively, appear to be sufficient to capture the most important features of the shell. However, in the case of Steromphala and Phorcus, shell morphometrics does not always serve species delineation without additional, nonmorphometric characters, like shell sculpture in Ph. mutabilis specimens or very distinct and stable colouration in St. adriatica. 


\section{Systematics: genus delineation and species diagnosis}

The molecular tree (Fig. 3) recovers a monophyletic Phorcus clade, but a paraphyletic situation for species traditionally placed in Gibbula, which agrees with earlier studies (Donald et al. 2012; Uribe et al. 2016; Williams et al. 2010; Williams 2012). We therefore propose the genus-group name Steromphala Gray, 1847 with the type species St. cineraria (Linnaeus, 1758) for this monophyletic clade and restrict the use of the name Gibbula to the group of its type species, G. magus (Linneaus, 1758).

Geometric shell morphometrics of the Mediterranean taxa serves well in separating Phorcus from Steromphala, supporting the recognition of Phorcus as a valid genus by Gofas and Jabaud (1997). The only exception is a Croatian specimen that genetically belongs to $P h$. mutabilis with a shell morphometrically resembling St. adriatica (Fig. 4). A closer inspection of the shell itself shows a clear distinction in shell size and colouration, as well as umbilical atrium morphology, which enable easy differentiation between these species.

Genus Steromphala Gray, 1847

p. 146, as junior synonym of Gibbula

Type species: Steromphala cineraria (Linneaus, 1758), by original designation

Steromphala is defined by a subtriangular shell shape and a concave base. Fine ridges and striae are present, but prominent nodes or cusps such as in Gibbula magus are not developed. The upper and lower aperture attachment points are separated by a quarter turn of the whorl or less $\left(\alpha \leq 90^{\circ}\right)$. The umbilicus is open and may be set off the shell base by an umbilical atrium. The only species closing the umbilicus with age is $S t$. divaricata.
Fig. 6 Steromphala umbilicaris (Linnaeus, 1758) and Steromphala nebulosa (Philippi, 1848). a PCA plot of PC1 vs. PC2 of genus Steromphala. Steromphala umbilicaris (green) separates well from all other species. There is no overlap between specimens from this study and the type material. One individual (Linné 24) nests within St. adriatica. Steromphala nebulosa (grey) nests at the edge of St. umbilicaris. b Representative specimen of St. umbilicaris from this study. c Representative specimen of St. nebulosa from this study. d: Designated lectotype for St. umbilicaris located at LSL (LSL.504). Scale bars $5 \mathrm{~mm}$
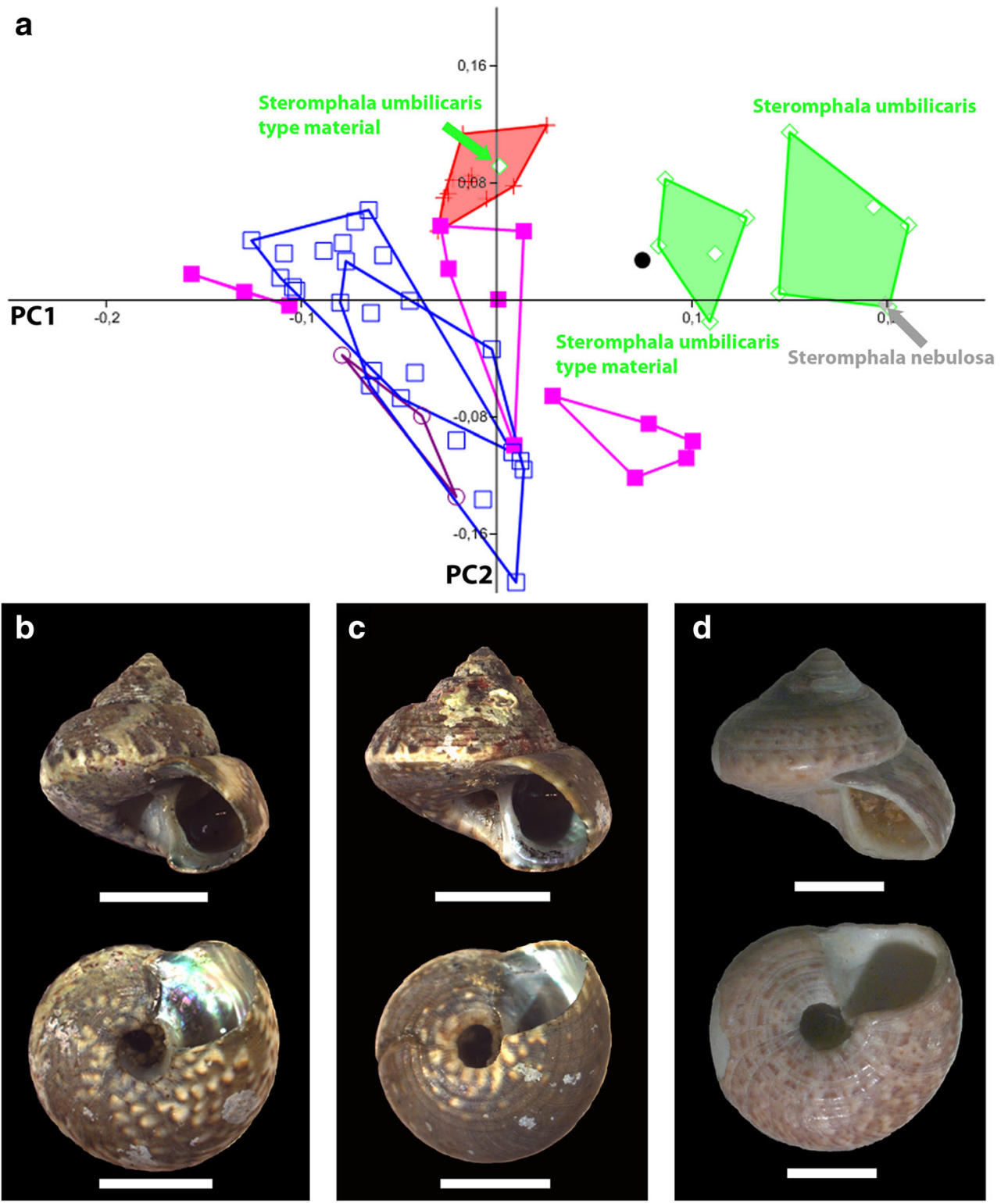

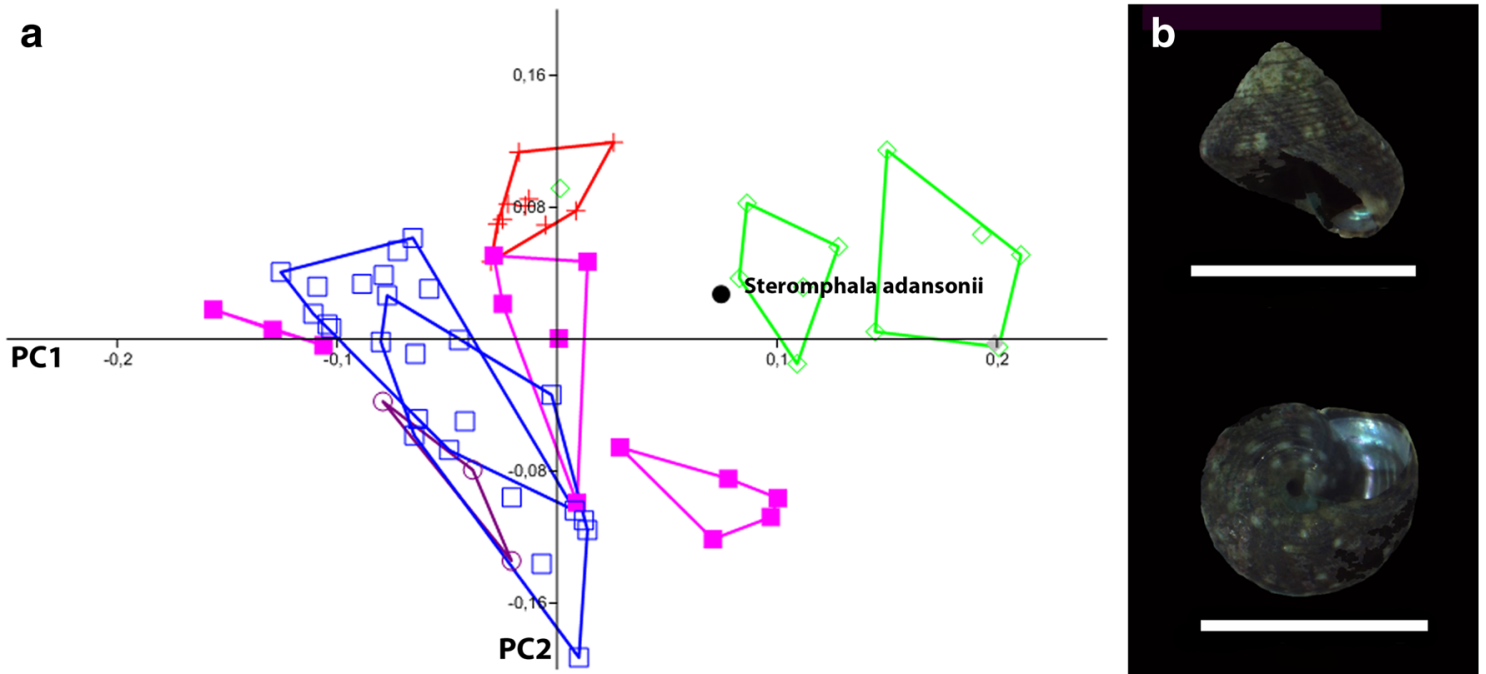

Fig. 7 Steromphala adansonii (Payraudeau 1826). a PCA plot of PC1 vs. PC2 of genus Steromphala. Single specimen of Steromphala adansonii (black) clusters near St. umbilicaris. b Representative specimen of St. adansonii from this study. Scale bar $5 \mathrm{~mm}$

Steromphala cineraria (Linnaeus, 1758)

Original combination: Trochus cinerarius Linnaeus, 1758: p 758, n 512.

Type material: LSL.502 (Linnean Society of London, UK), ten specimens.

We here designate the specimen "Linné 35" (this shell is marked " 512 ," corresponding to the number given in the original description), as lectotype (Fig. 5). The remaining nine type specimens are paralectotypes.

Steromphala cineraria was originally described as an umbilicated shell with rounded whorls. Its overall shape is triangular. The sculpture consists of distinct spiral ridges and the small umbilical atrium has a sharp edge. The base is concave with a narrow and round umbilicus.

Steromphala umbilicaris (Linnaeus, 1758)

Original combination: Trochus umbilicaris Linnaeus, 1758: p 758, n 514

Type material: LSL.504 (Linnean Society of London, UK), six specimens.

We here designate the specimen "Linné 23" (Fig. 6d) as lectotype. The remaining five specimens are paralectotypes. (The identification of specimen "Linné 24" as St. umbilicaris is questionable.)

The original description of this species refers to an open and deep cylindrical umbilicus, white in colour. The whorls
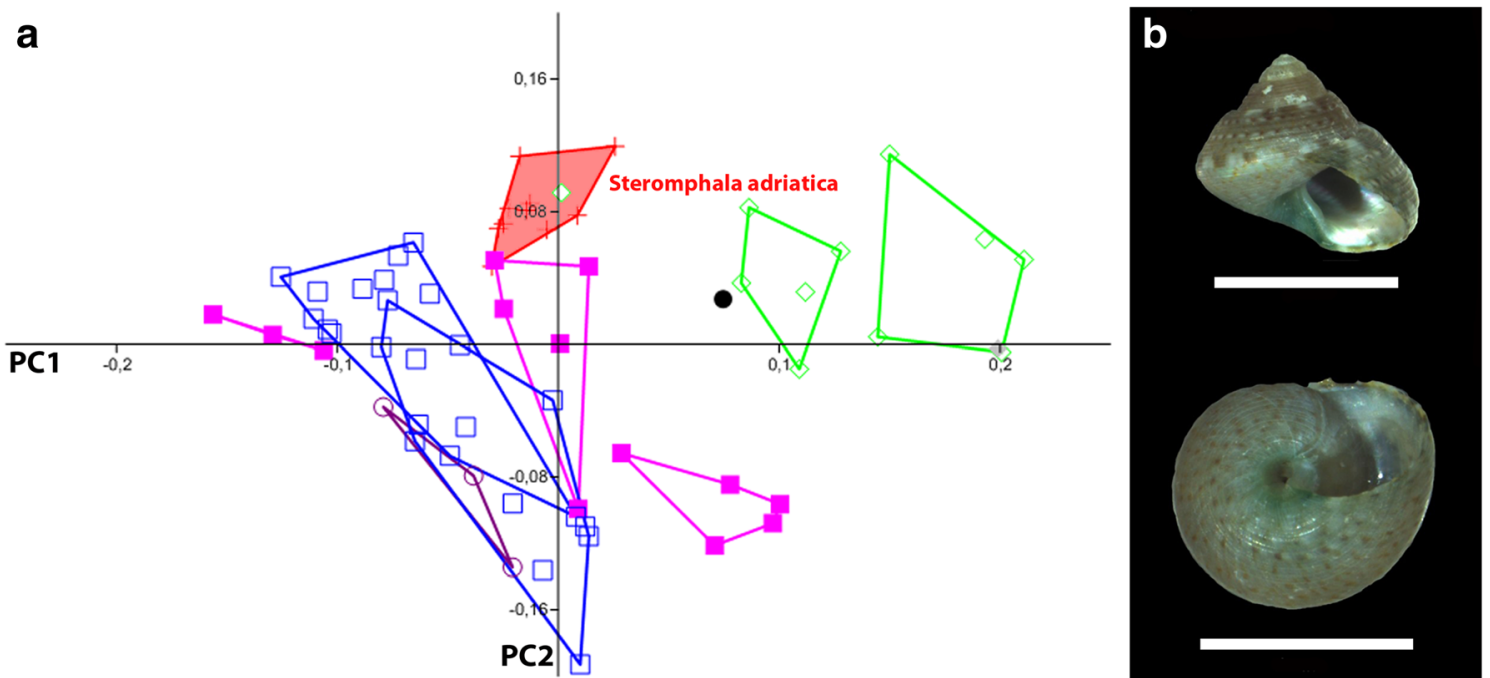

Fig. 8 Steromphala adriatica (Philippi, 1844). a PCA plot of PC1 vs PC2 of genus Steromphala. Steromphala adriatica (red) is separated from all but St. divaricata. One individual of Steromphala umbilicaris (Linné
24) nests within St. adriatica. b Representative individual of St. adriatica from this study. Scale bar $5 \mathrm{~mm}$ 

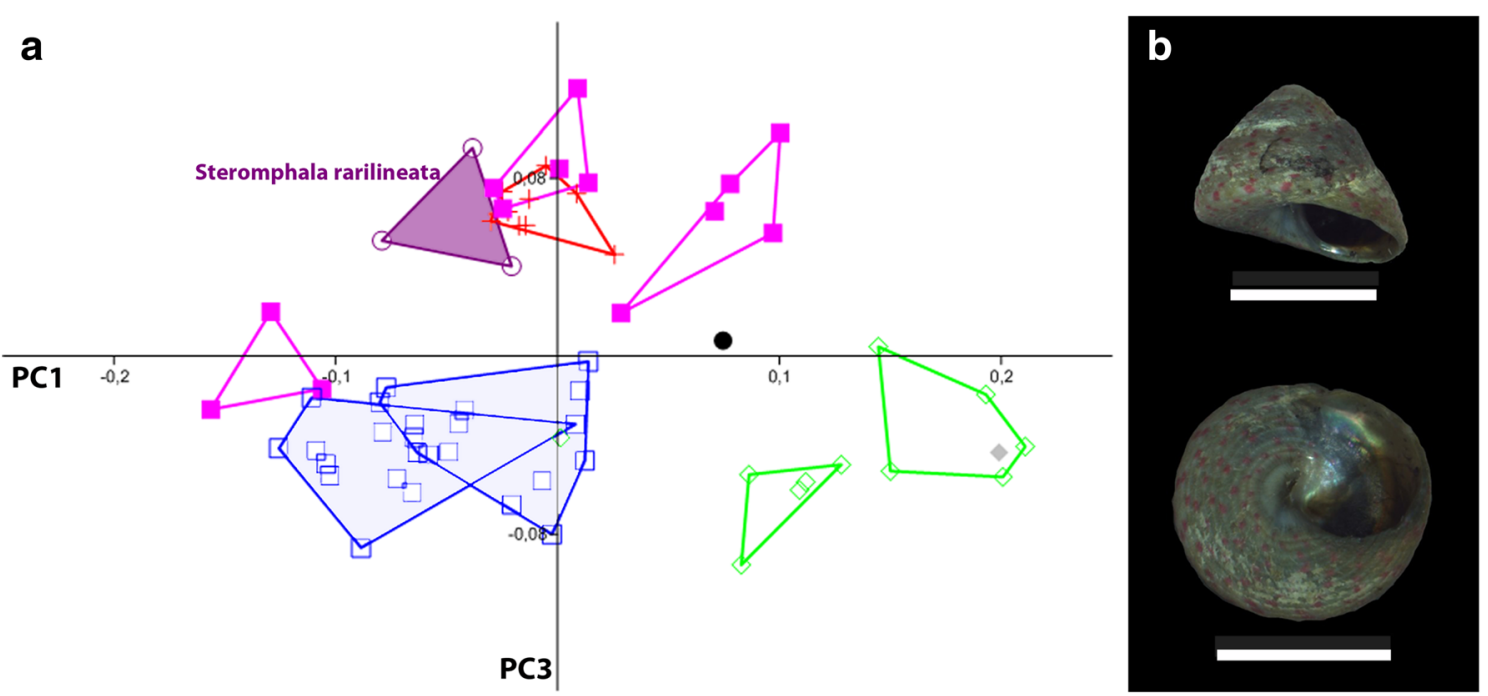

Fig. 9 Steromphala rarilineata (Michaud, 1829). a PCA plot of PC1 vs. PC3 from genus Steromphala. Steromphala rarilineata (violet) is separated but very near St. adriatica and St. divaricata. b Representative specimen of St. rarilineata from this study. Scale bar $5 \mathrm{~mm}$

are conic and convex with a shallow suture. The individuals collected for this study show a matching deep and cylindrical umbilicus, but the whorls are shouldered and separated by an incised suture (e.g. Fig. 6b). The type material consists of six shells with a wide variety of shapes, none of which resembles our specimens, and only one shows convex whorls as described by Linnaeus. We therefore designate this individual as lectotype (Linnè 23, Fig. 6d). Steromphala umbilicaris sometimes shows a slightly convex shell base. The umbilicus is deep and round. Its columella base is angular. The shell diameter is bigger than the shell height. The aperture is small, with the attachment points only separated by approximately an eighth of a shell turn $\left(\alpha \approx 45^{\circ}\right)$.
The wide range of variability in shell shape resembles that of Steromphala divaricata. As there is no information on the type locality, future investigations may address potential geographic differentiation of shell shape in these species.

Steromphala nebulosa (Philippi, 1849)

Original combination: Trochus nebulosus Philippi, 1849: $\mathrm{p}$ 109-110, n 32

Type material not located.

DNA-barcoding suggests the distinction of the sister-species Steromphala nebulosa and St. umbilicaris (Barco et al. 2013). The molecular analysis in this study further strengthens the argument for the validity of both species. The split between St. umbilicaris and St. nebulosa is well supported (bootstrap
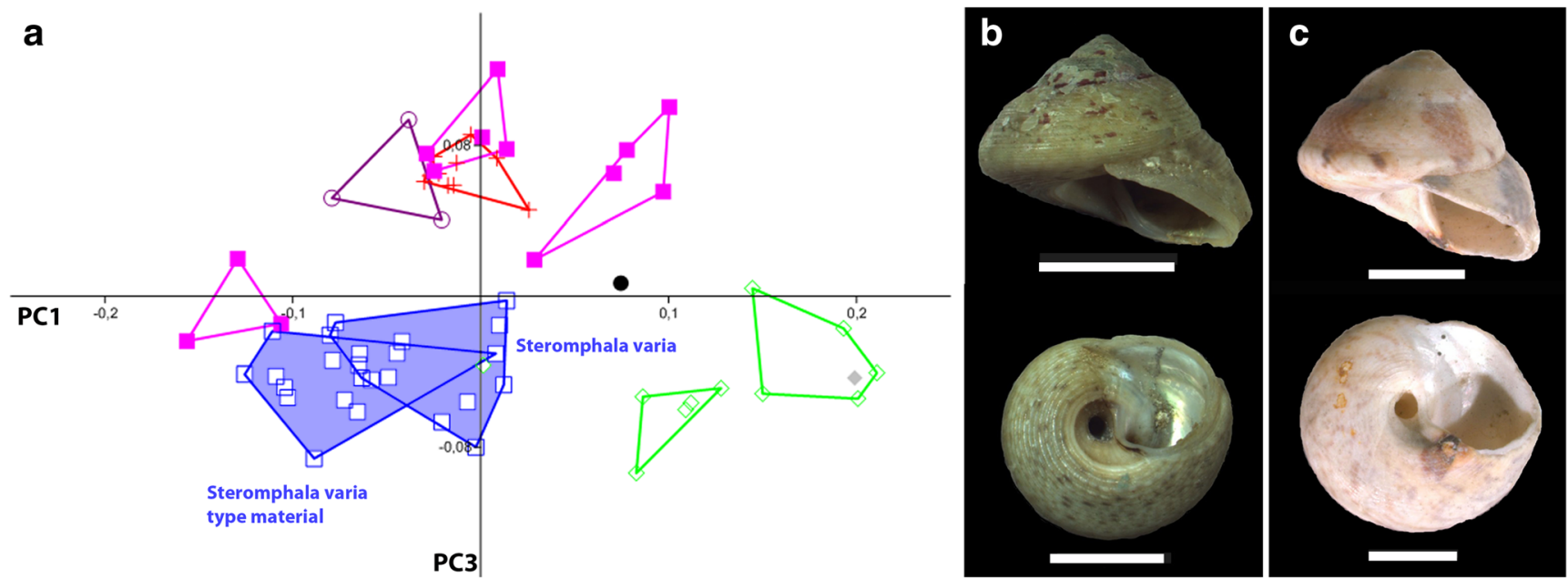

Fig. 10 Steromphala varia (Linnaeus, 1758). a PCA plot of PC1 vs. PC3 of genus Steromphala. Steromphala varia (blue) separates from all other species. Specimens from this study overlap with the type material. b

Representative specimen of St. varia from this study. c Lectotype designated for St. varia (LSL.501). Scale bars $5 \mathrm{~mm}$ 

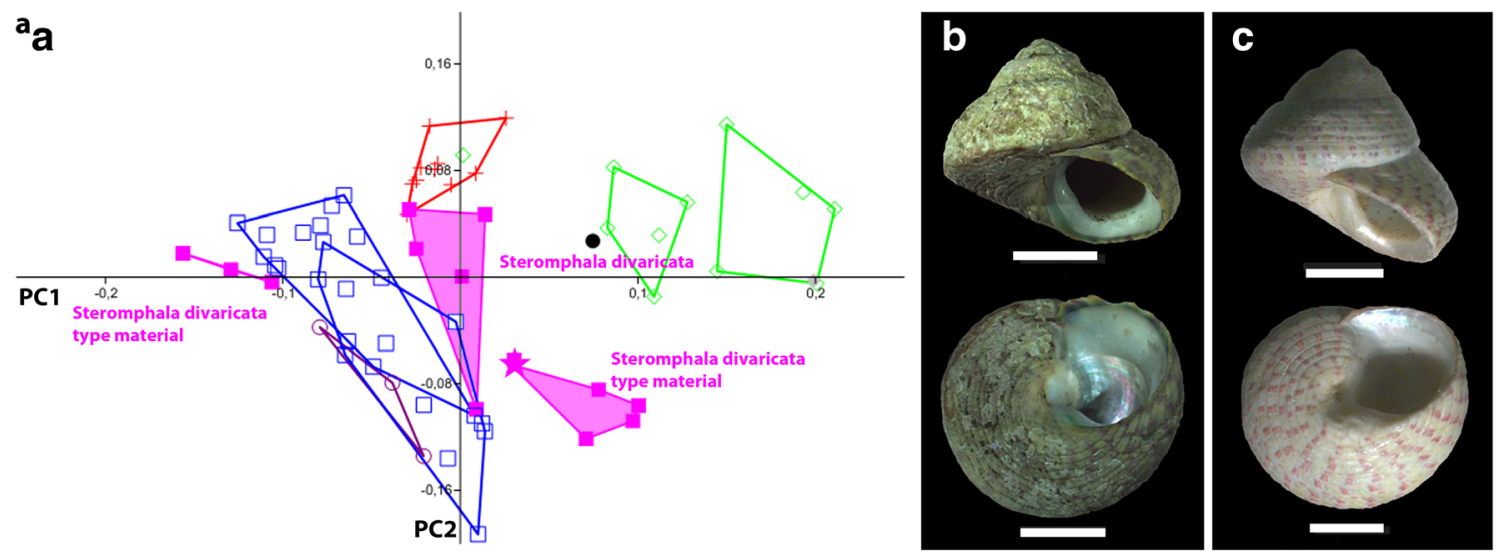

Fig. 11 Steromphala divaricata (Linnaeus, 1758). a PCA plot of PC1 vs. PC2 from genus Steromphala. Steromphala divaricata (pink) cannot be recovered as a completely separated group as it shows a small overlap with St. varia. Type material and material from this study do not overlap. Three individuals (Linné 41-43) of the type material (pink group on the

value $=100$ ). Specimens of both species were sampled from Italy and Croatia, and the tree does not show a geographical bias, corroborating the separation of the two taxa. However, the specimens barcoded for each species in this study cannot be distinguished morphologically. The single specimen identified as St. nebulosa by COI barcoding following Barco et al. (2013) nests within St. umbilicaris in the morphometric analyses (Figs. 6a and 9a). A re-examination of the shell itself did not yield any distinguishing characters.

The original description of St. nebulosa reports an umbilicated conical shell, reddish with white marks; whorls are convex, with the last one being slightly angular. Philippi (1849) mentioned six sulci on the whorls and four striae at the base. Although this description is more detailed than that of Steromphala umbilicaris, it fails to mention diagnostic differences. As none of the collected specimens (Fig. 6c) overlap left) were re-identified as St. varia (compare Fig. 8). b Representative specimen of St. divaricata from this study. c Lectotype (pink star) designated for St. divaricata (LSL.503) by Anistratenko and Anistratenko (2001). Scale bars $5 \mathrm{~mm}$

with the available type material in the geometric morphometric analysis or matches one of the original descriptions sufficiently to differentiate between the two species, morphological definitions of St. umbilicaris and St. nebulosa remain questionable. From a conchological point of view, these two species have to be defined as cryptic sister species. Further morphological investigations, e.g. radula comparison, and a larger molecular sample are necessary to determine if they are truly cryptic.

Steromphala adansonii (Payraudeau 1826)

Original combination: Trochus adansonii Payraudeau 1826: p127, n 267, Pl. 6, Figs. 7 and 8.

Type material: MNHN-IM-2000-30071 (Muséum national d'Histoire naturelle, Paris, France), 3 syntypes.

The original description of Steromphala adansonii focuses on the colouration of the shell. Payraudeau (1826) describes
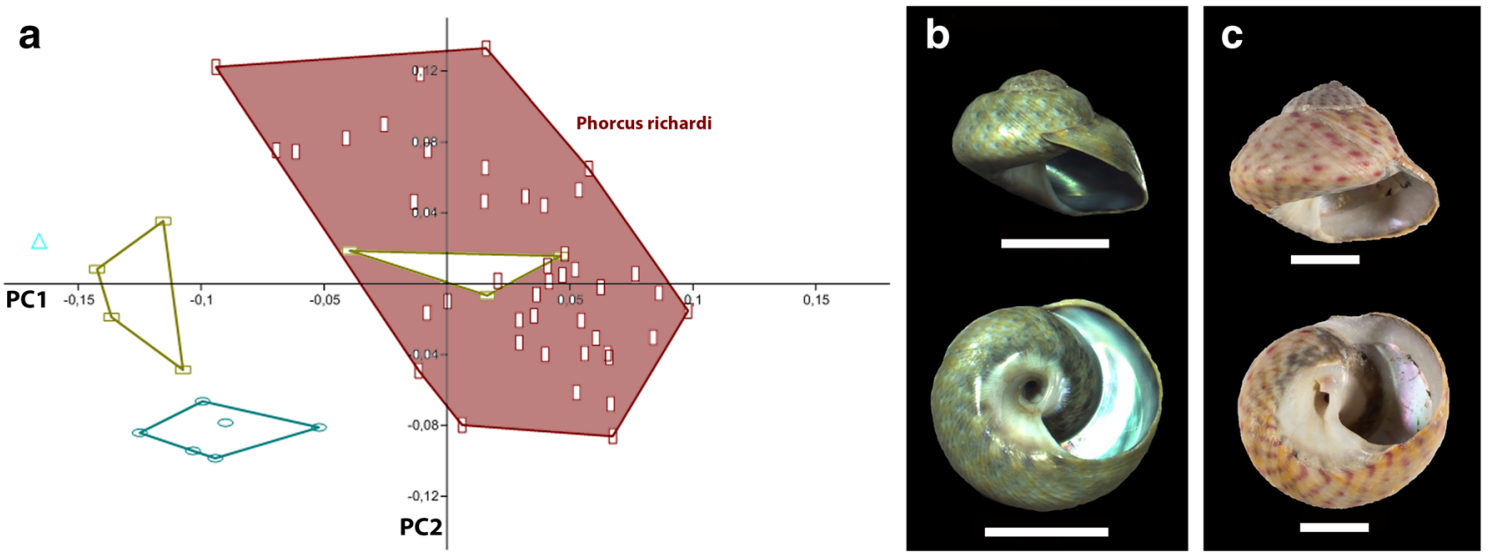

Fig. 12 Phorcus richardi (Payraudeau 1826). a PCA plot of PC1 vs. PC2 of genus Phorcus. Phorcus richardi (maroon) is the most abundant species in the current sample. It separates well from $P h$. articulatus and $P h$. turbinatus. One group of $P h$. mutabilis is not distinguishable from $P h$.

richardi in the first two principle components. b Representative specimen of $P h$. richardi from this study. $\mathbf{c}$ One syntype of $P h$. richardi. Located at MNHN (MNHN-IM-2000-28253). Scale bars 5 mm 
Fig. 13 Phorcus mutabilis (Philippi, 1846). a PCA plot of PC1 vs. PC3 from genus Phorcus. Phorcus mutabilis (olive) splits into two morphotypes: one from Croatia and one from Crete. $\mathbf{b}$ Representative specimen of $P h$. mutabilis (Croatia) from this study. c Representative specimen of Ph. mutabilis (Crete) from this study. d Specimen (black arrow) showing $P h$. richardi morphology, but Ph. mutabilis COI barcode. From this study. Scale bars $5 \mathrm{~mm}$
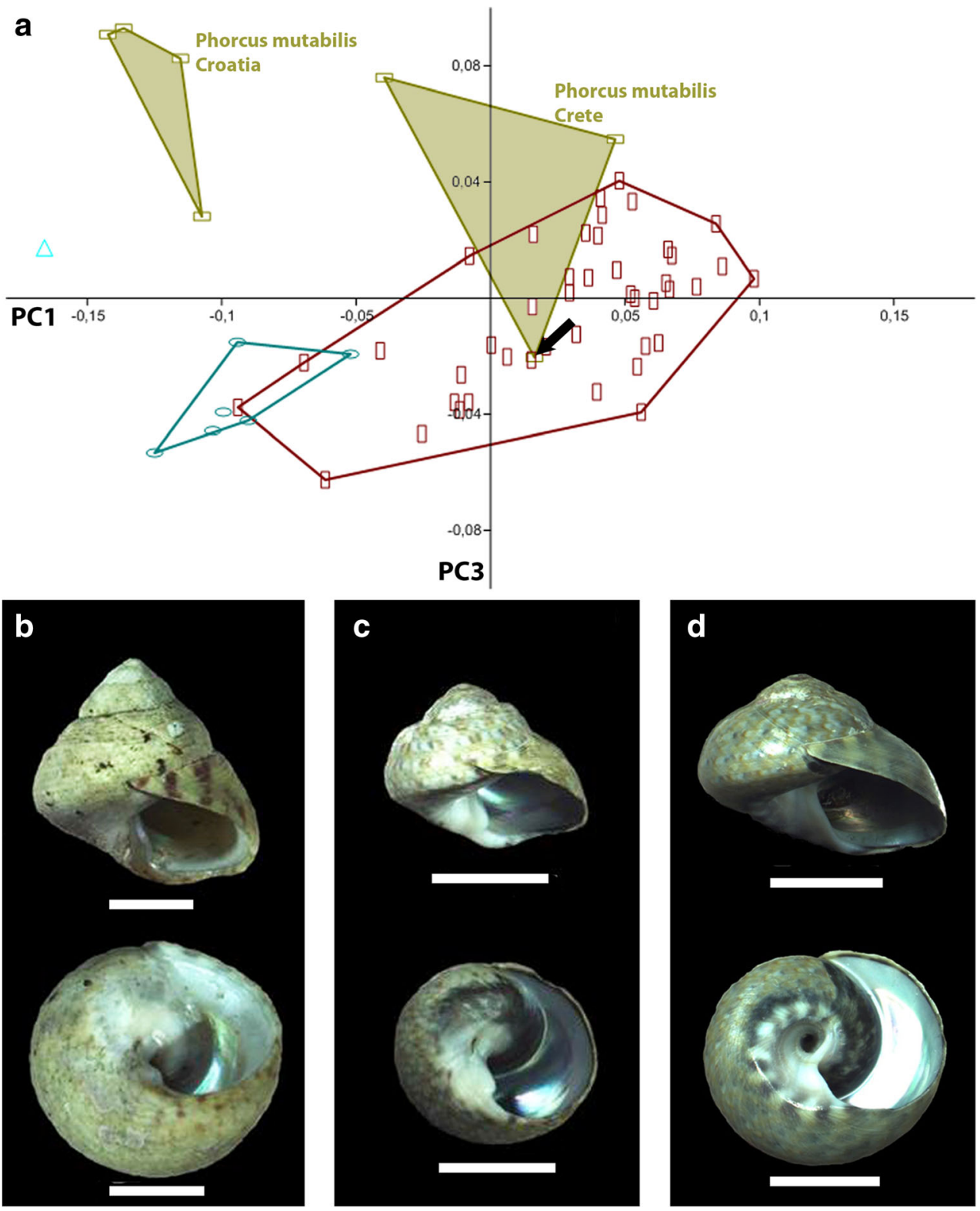

Originally described as a variation of Steromphala adansonii, it differs from this species by the angular suture of the bigger last whorl. Philippi (1844) mentions a node on the columellar side of the aperture, but this is not observed in the present specimens. Morphometric and genetic data confirm the separation of the sister species, St. adriatica and St. adansonii.

Steromphala adriatica shows a distinct shape in lateral and ventral views. It is characterised by the larger last whorl, an acute apex, as well as a narrow, round, and conical umbilicus without distinct atrium. The whorls are slightly shouldered. Fresh shells of this species always show green colouration around the umbilicus, which tends to fade in dry and preserved shells. The sculpture consists of several spiral lines, sometimes marked in colour or white bands (Fig. 6).
Steromphala adriatica (Philippi, 1844)

Original combination: Trochus adriaticus Philippi, 183644: p 153, n 21, Tab. XXV, Fig. 10

Type material not located. 

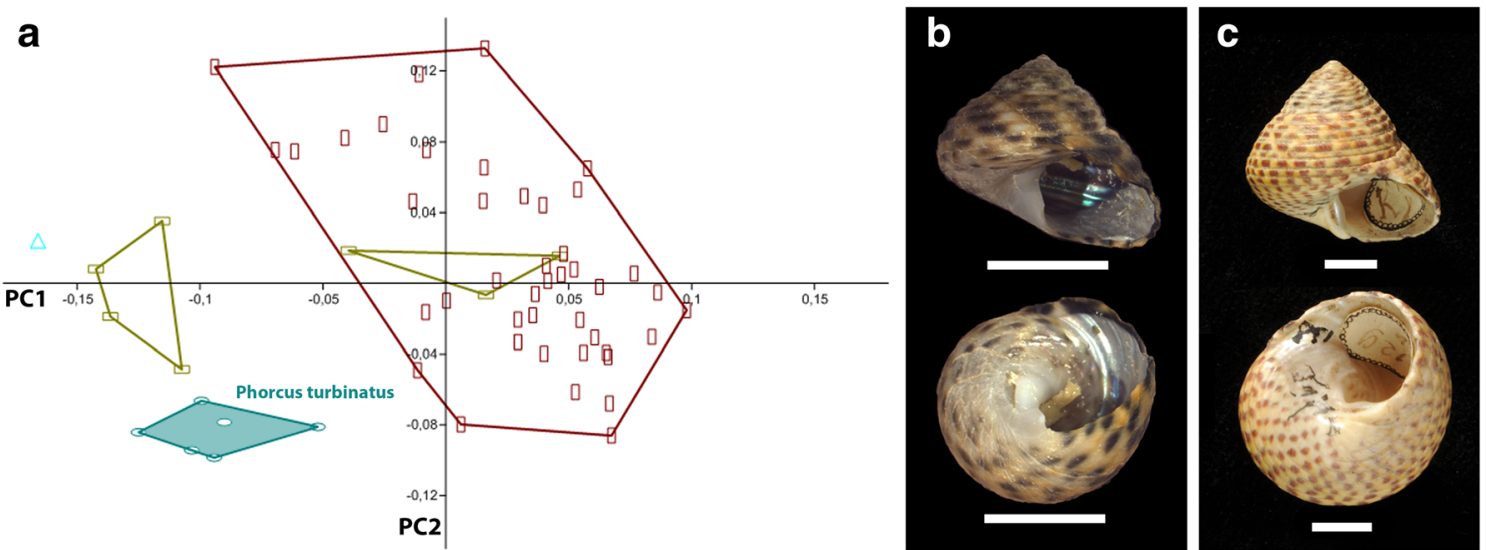

Fig. 14 Phorcus turbinatus (Born, 1778). a PCA plot of PC1 vs. PC2 for genus Phorcus. Phorcus turbinatus (petrol) separates from all other species. b Representative specimen of Ph. turbinatus from this study. c One syntype of Ph. turbinatus (NHMW 14002). Scale bars $5 \mathrm{~mm}$

Steromphala rarilineata (Michaud, 1829)

Original combination: Trochus rarilineatus Michaud, 1829: p 266-267, n 8, Fig. 12

Type material: lot ${ }^{\circ} 45011450$ (Collection Malacologie, Musée des Confluences, Lyon, France), three syntypes (compare Boyer and Audibert 2007). Type material was not re-examined for this study.

Although this species cannot be distinguished from St. varia in the first two principal components (Fig. 8a), it is recovered in the third (Fig. 9a). Compared to St. varia, it differs in the shape of the umbilicus and the absence of an umbilical atrium (Fig. 9b). A distinction between St. rarilineata and juveniles of St. divaricata is very difficult. In agreement with the original description of this species, the typical shell characters include angular whorls with indistinct suture and multiple curved lines of reddishpurple dots on the fine shell sculpture. The colouration pattern is very common but unreliable as a diagnostic feature, because it is shared with St. divaricata and disappears in dry material or shells stored in ethanol.

Steromphala rarilineata is defined by an overall triangular shape. Its diameter and height are nearly the same. The base is slightly concave. The aperture lip is strongly tilted and attaches exactly on the angular lower edge of the last whorl, leaving the shell with no distinct suture (Fig. 9b).

\section{Steromphala varia (Linnaeus, 1758)}

Original combination: Trochus varius Linnaeus, 1758: $\mathrm{p}$ 758, n 511

Type material: LSL.501 (Linnean Society of London, UK) 14 specimens.

We here designate the specimen "Linné 7" as lectotype (Fig. 10c). The 13 remaining specimens are paralectotypes.

Steromphala varia is originally described as a shell with an overall convex shape and an open umbilicus. Linnaeus did not mention the diagnostic umbilical atrium. The collected specimens correspond well with the type material.

Steromphala varia is the only species with a well-defined, sharp-edged umbilical atrium and a wide and round umbilicus (compare Gofas et al. 2011) (Fig. 10). Furthermore, St. varia shows several spiral ridges and a more or less visible suture. The shell base is slightly concave. The aperture is tilted and the lip attaches just below the angle of the last whorl.
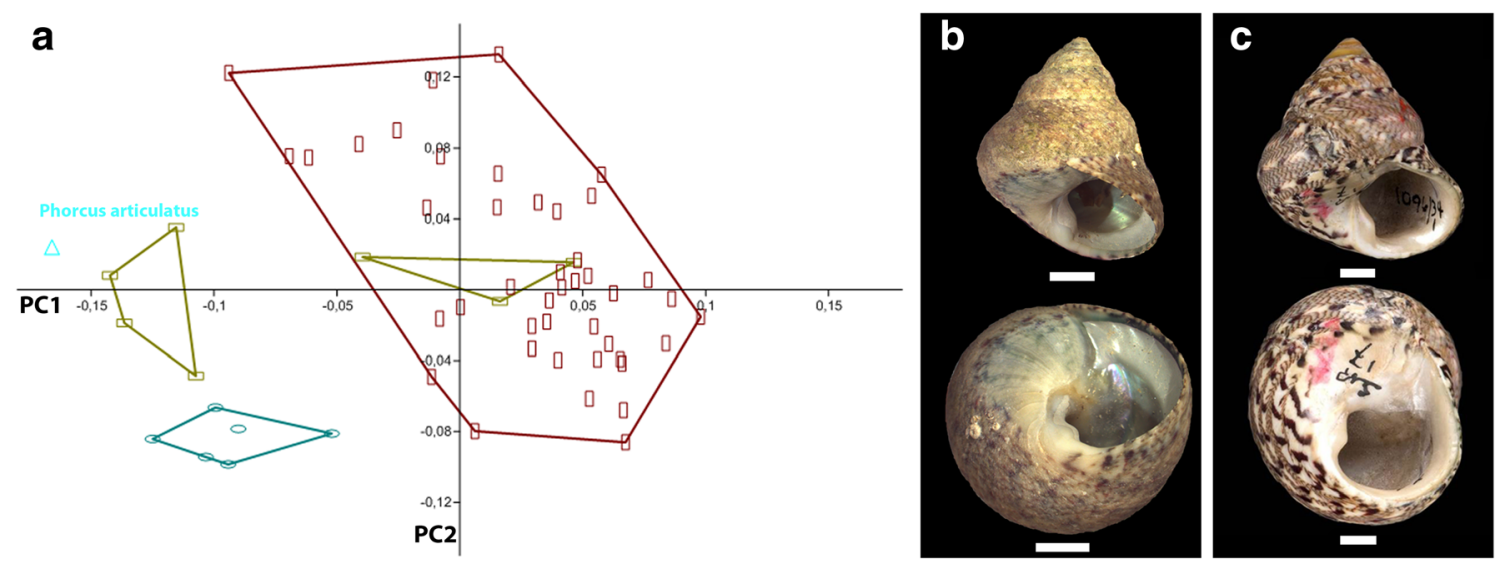

Fig. 15 Phorcus articulatus (Lamarck 1822). a PCA plot of PC1 vs. PC2 for genus Phorcus. Single specimen of Phorcus articulatus separates from all other species. b Representative specimen of Ph. articulatus from this study. c One syntype of Ph. articulatus (INVE 51532). Scale bars $5 \mathrm{~mm}$ 
Steromphala divaricata (Linnaeus, 1758)

Original combination: Trochus divaricatus Linnaeus, 1758: p 758, n 513

Type material: LSL.503 (Linnean Society of London, UK), eight specimens.

Lectotype (Fig. 11c) and seven paralectotypes (Anistratenko and Anistratenko 2001, English translation: Anistratenko 2005)

The great variability in shell shape makes Steromphala divaricata difficult to identify (Fig. 11a). The original species description mentions an ovate shell with a mostly closed umbilicus and deep suture. The set-off last whorl, however, is not always developed as already observed by Anistratenko and Anistratenko (2001) (Anistratenko 2005). The specimens examined here vary from angular whorls (Fig. 11b) reminiscent of St. varia and St. rarilineata, to convex whorls as mentioned in the original description. But regardless of the variation in the lateral aspect and the overall size of the shell, all individuals showed a slit-like umbilicus and no distinct umbilical atrium. Future studies may shed light on potential geographic variation and ecophenotypic plasticity.

The examination of the type material revealed three of the current paralectotypes (Linné 41- Linné 43) belonging to St. varia and need to be excluded from the type material of $S t$. divaricata (Figs. 10a and 11a).

A shell with a larger last whorl characterises Steromphala divaricata. The aperture lip attaches below the edge of the last whorl, which can be round or angular, and leaves the shell with a distinct suture. The aperture is small and round. The shell base is flat. The umbilicus is slit like or completely closed by the columellar fold. There is no umbilical atrium.

\section{Genus Phorcus Risso, 1826}

Type species: Phorcus margaritaceus Risso, 1826 (by subsequent designation), accepted as Phorcus richardi (Payraudeau 1826)

Phorcus is characterised by a subtriangular shell shape with whorls more rounded than in Steromphala and a convex base. The shells are either smooth or show coarser spiral striae than in Steromphala. The umbilicus narrows with age. In large species, it can be closed completely by a columellar fold. The upper and lower aperture attachment points are separated by at least a quarter turn of the whorl $\left(\alpha \geq 90^{\circ}\right)$.

Phorcus richardi (Payraudeau 1826)

Original combination: Monodonta richardi Payraudeau 1826: p 138, n278, Pl VII, Figs. 1 and 2.

Type material: MNHN-IM-2000-28253 (Muséum National d'Histoire naturelle Paris, France), two syntypes.

The shell of Phorcus richardi is depressed with a bloated last whorl as originally described (Payraudeau 1826). It appears rather thin-walled, and the inside of the rhomboid aperture always shows a layer of bluish nacre (Gofas et al. 2011;
Nordsieck 1968; Payraudeau 1826) (Fig. 12). The type material matches the original descriptions well and corresponds to the collected specimens (Fig. 12).

Phorcus richardi is characterised by a distinct shell shape and an open, large, round umbilicus surrounded by a rounded, white umbilical atrium edged by a black and white pattern, which is unique for this species (compare Gofas et al. 2011; Nordsieck 1968). The shell is wider than high. The attachment points of the aperture are separated by nearly a half turn of the shell $\left(\alpha \approx 180^{\circ}\right)$. The aperture lip attaches above the whorl edge with a slight shoulder and suture.

Phorcus mutabilis (Philippi, 1846)

Original combination: Trochus mutabilis Philippi, 1846: $\mathrm{p}$ 166, n 201, Pl 26, Figs. 18-22

Type material not located.

The original description by Philippi (1846) distinguishes Phorcus mutabilis from Ph. turbinatus by the denticle at the base of the columella. This denticle is not present in all individuals identified as Ph. mutabilis by DNA-barcoding of the present material. One of the specimens genetically identified as $P h$. mutabilis shows the typical shell shape of $P h$. richardi (Fig. 13a and d, black arrow). Future research including nuclear genetic markers could elucidate possible hybridisation or incomplete lineage sorting in these species. Furthermore, $P h$. mutabilis shows two distinct morphotypes: the individuals from Crete closely resemble Ph. richardi (Fig. 13c) in shell shape; two of the Crete specimens (e.g. Fig. 13c), however, have the columellar denticle and spiral lines typical for $P h$. mutabilis but not for $P h$. richardi (compare Philippi 1846). Specimens from Croatia rather resemble Ph. turbinatus (Figs. $13 \mathrm{~b}$ and 14), a similarity already mentioned in the original description. They also show similarities to Ph. articulatus (Fig. 15).

In all Phorcus mutabilis specimens examined, the umbilicus is not as open as in Ph. richardi. It seems as if the umbilicus becomes smaller or is even completely closed as the shell grows (compare Philippi 1846).

Further research is needed to understand the mechanisms behind this high variability in shell morphology.

Phorcus turbinatus (Born, 1778)

Original combination: Trochus turbinatus Born, 1778: 340-341, n K.II.4

Type material: NHMW14002 (Naturhistorisches Museum Wien, Austria), two syntypes.

Phorcus turbinatus is originally described having an ovate shell with smooth whorls and a denticle at the columellar base. The specimens collected for this study match the original description and the type material (Fig. 14c) in their overall shape and the lack of an umbilicus, which is closed by a columellar fold. They differ, however, in lacking a denticle, and the presence of prominent spiral ridges (Fig. 14b). These differences 
may be due to the relatively small size of the collected shells. They seem to be immature and probably lose the shell sculpture with age.

Phorcus turbinatus shows a compact shell with a convex base. The slit like umbilicus is closed with age by a columellar fold. The aperture lip attaches on the round whorl edge, leaving a small shoulder and shallow suture.

\section{Phorcus articulatus (Lamarck 1822)}

Original combination: Monodonta articulata de Lamarck 1822: p 36, n 17

Type material: INVE 51532 (Muséum d'Histoire naturelle Genéve, Switzerland), four syntypes.

Phorcus articulatus is originally described as having a conical shell with very convex whorls. The umbilicus, round and small, is closed with age. The columella base is marked by a distinct, rounded denticle. The single specimen of $P h$. articulatus in this study (Fig. 15b), as well as the syntypes (e.g. Fig. 15c), matches the original description.

The shell shape of Phorcus articulatus is characterised by a very high spiral height to diameter ratio. The columella shows a distinct denticle. The aperture lip attaches below the rounded whorl edge, leaving each whorl bulbous and without shoulder or suture. The aperture is comparatively small for Phorcus species, with attachment point set apart by just a quarter of a shell turn $\left(\alpha \approx 90^{\circ}\right)$.

\section{Conclusion}

Robust molecular phylogenetic results showing Gibbula as a paraphyletic group relative to Phorcus are calling for a taxonomic reassessment. Both COI barcoding and geometric morphometric analyses underscore the separation of the former subgenus of Gibbula, Steromphala Gray, 1847, from Phorcus Risso, 1826 and Gibbula s.s. Risso, 1826, and its elevation to full genus rank. Shell shape is an informative and reliable character to distinguish between Steromphala and Phorcus species significantly aiding identification even in the field.

More subtle differences in shell shape and the intraspecific variability make species identification within Steromphala and Phorcus difficult with the naked eye, even if most species are characterised by shell features. These species-specific shell shapes can only be resolved by combining lateral and ventral views with their information on the orientation of the aperture and umbilicus features. Thus, geometric morphometrics is a powerful tool to aid identification of most species in this study. It is also a non-destructive method for assessing type material or fossils. In combination with DNA-barcoding on recent material, it offers the possibility of objectively linking dry material to molecular markers. Although (morphologically trained) human vision is a powerful tool in separating morphotypes, gastropod shells with contour dissolving colourations can pose problems. Geometric morphometrics capture the true shape of the shell, regardless of colour and pattern. It can therefore show similarities among species otherwise perceived as very different due to colourations, intraspecific variability, or ontogenetic shapeshifts. Although it is not as easily applicable as classical morphometrics, e.g. length measurements, it serves significantly better in separating taxa in the present study.

However, the spatial resolution of sliding landmarks may be too low to detect some of the smaller shell features like the aperture denticle in Ph. mutabilis. Alternative methods such as outline analysis or a more narrow set of fixed landmarks could potentially overcome this problem. The problem of defining homologous landmarks on gastropod shells is a limiting factor.

Further analyses including nuclear genes and/or microsatellites on a broader taxon sample are required for resolving the internal relationships in the Gibbula-SteromphalaPhorcus group.

Some species, like Steromphala nebulosa, need further morphological investigations. Ongoing research on the remaining syntypes will result in the designation of additional lectotypes and, in the cases of missing type material, neotypes for the remaining Gibbula, Steromphala, and Phorcus species. The clarification of ambiguous type material, like in $S t$. divaricata, will continue with further geometric morphometric analyses and appropriate sampling of live specimens in combination with molecular barcodes for taxon assignment and the designation of epitypes (Schrödl and Haszprunar 2016).

Acknowledgements Open access funding provided by University of Vienna. We are indebted to Andreas Traxler for hosting the collecting trip to Crete and for generously providing our operational base. Michel Bariche and Ahmad Jammal (American University Beirut) provided invaluable help while collecting in Lebanon. We would like to thank the curators, Kathie Way (Linnean Society of London Collection), Emmanuel Tardy (Museum of Natural History Geneva), Virginie Herós (Musée National d'Histoire Naturelle Paris), Andreia Salvador (Natural History Museum London), Anita Eschner (Natural History Museum Vienna), and Erica Mejlon (Zoological Museum at University of Uppsala) for kindly providing information, pictures, and help with the inspection of type material. We would like to thank two anonymous reviewers for their insights and suggestions, which greatly improved the quality of the manuscript.

Open Access This article is distributed under the terms of the Creative Commons Attribution 4.0 International License (http:// creativecommons.org/licenses/by/4.0/), which permits unrestricted use, distribution, and reproduction in any medium, provided you give appropriate credit to the original author(s) and the source, provide a link to the Creative Commons license, and indicate if changes were made.

\section{References}

Anistratenko, V. V. (2005). Lectotypes for Tricolia pullus, Gibbula divaricata and Theodoxus fluviatilis (Mollusca, Gastropoda) revisited. Vestnik zoologii, 39(6), 3-10. 
Anistratenko, V. V. \& Anistratenko, O. Y. (2001). Mollusca. Class Polyplacophora or chitons, Class Gastropoda-Cyclobranchia, Scutibranchia and Pectinibranchia (part). Fauna Ukrainy, 29(1), 1240.

Bálint, M., Domisch, S., Engelhardt, C. H. M., Haase, P., Lehrian, S., Sauer, J., Theissinger, K., Pauls, S. U., \& Nowak, C. (2011). Cryptic biodiversity loss linked to global climate change. Nature Climate Change, 1, 313-318.

Barco, A., Evans, J., Schembri, P. J., Taviani, M., \& Oliverio, M. (2013). Testing the applicability of DNA barcoding for Mediterranean species of top-shells (Gastropoda, Trochidae, Gibbula s.l.) Marine Biology Research, 9(8), 785-793.

Barco, A., Raupach, M. J., Laakmann, S., Neumann, H., \& Knebelsberger, T. (2016). Identification of North Sea molluscs with DNA barcoding. Molecular Ecology Resources, 16(1), 288-297.

Bookstein, F. L. (1991). Morphometric tools for landmark data: geometry and biology. Cambridge University Press.

Bookstein, F. L. (1996). Biometrics, biomathematics and the morphometric synthesis. Bulletin of Mathematical Biology, 58(2), 313-365.

Bookstein, F. L. (1997). Landmark methods for forms without landmarks: Morphometrics of group differences in outline shape. Medical Image Analysis, 1(3), 225-243.

von Born, I. (1778). Index rerum naturalium Musei Casarei Vindobonensis. Pars I.ma. Testacea. Verzeichniß der natürlichen Seltenheiten des $k$. $k$. Naturalien Cabinets zu Wien. Erster Theil. Schalthiere. Wien: Kraus.

Boyer, F., \& Audibert, C. (2007). Le Matériel d'auteur conservé au Muséum de Lyon pour les Taxa de Michaud, 1828 et 1829. Cahiers scientifiques-Muséum d'histoire naturelle de Lyon, 13, 149-159.

Delicado, D., \& Ramos, M. A. (2012). Morphological and molecular evidence for cryptic species of springsnails [genus Pseudamnicola (Corrosella) (Mollusca, Caenogastropoda, Hydrobiidae)]. ZooKeys, 190, 55-79.

Donald, K. M., Kennedy, M. \& Spencer, H. G. (2005). Cladogenesis as the result of long-distance rafting events in South Pacific topshells (Gastropoda, Trochidae). Evolution, 59(8), 1701-1711.

Donald, K. M., Preston, J., Williams, S. T., Reid, D. G., Winter, D., Alvarez, R., Buge, B., Hawkins, S. J., Templado, J., \& Spencer, H. G. (2012). Phylogenetic relationships elucidate colonization patterns in the intertidal grazers Osilinus Philippi, 1847 and Phorcus Risso, 1826 (Gastropoda: Trochidae) in the northeastern Atlantic Ocean and Mediterranean Sea. Molecular Phylogenetics and Evolution, 62(1), 35-45.

Feckler, A., Zubrod, J. P., Thielsch, A., Schwenk, K., Schulz, R., \& Bundschuh, M. (2014). Cryptic species diversity: an overlooked factor in environmental management? Journal of Applied Ecology, 51(4), 958-967.

Folmer, O., Hoeh, W. R., Black, M. B., \& Vrijenhoek, R. C. (1994). Conserved primers for PCR amplification of mitochondrial DNA from different invertebrate phyla. Molecular Marine Biology and Biotechnology, 3, 294-299.

Gofas, S., \& Jabaud, A. (1997). The relationships of the Mediterranean trochid gastropods 'Monodonta' mutabilis (Philippi, 1846) and 'Gibbula' richardi (Payraudeau 1826). Journal of Molluscan Studies, 63(1), 57-64.

Gofas, S., Moreno, D., \& Salas, C. (2011). Moluscos Marinos de Andalucia Volumen I - Introducción General, Clase Solenogastres, Clase Caudofoveata, Clase Polyplacophora Y Clase Gastropoda (Prosobranchia). Málaga: Universidad de Málaga Servicio de Publicaciones e Intercambio Científico.

Gray, J. E. (1847). A list of the genera of recent Mollusca, their synonyms and types. Proceedings of the Zoological Society of London, 15, 146.
Hammer, Ø., Harper, D. A. T., \& Ryan, P. D. (2001). PASTPalaeontological statistics software package for education and data analysis. Paleontologia Electronica, 4(1), 9pp.

Kendall, D. G. (1981). The statistics of shape. In V. Barnett (Ed.), Interpreting multivariate data (pp. 75-80). New York: Wiley.

Kendall, D. G. (1984). Shape manifolds, procrustean metrics, and complex projective spaces. Bulletin of the London Mathematical Society, 16(2), 81-121.

Kirchner, S., Harl, J., Kruckenhauser, L., Duda, M., Sattmann, H., \& Haring, E. (2016). Phylogeography and systematics of Pyramidula (Pulmonata: Pyramidulidae) in the eastern Alps: still a taxonomic challenge. Journal of Molluscan Studies, 82(1), 110-121.

de Lamarck, J.-B. M. (1822). Histoire naturelle des animaux sans vertèbres. Tome septième. Paris: Author.

Linnaeus, C. (1758). Systema Naturae, Ed. 10, Vol. 1. 824pp. Salvii, Holmiae.

Michaud, A. L. G. (1829). Description de Plusieurs Espèces Nouvelles de Coquilles Vivantes. Bulletin d'histoire naturelle de la Societe Linneenne de Bordeaux, t., 3, 266-267.

Mitteroecker, P., \& Gunz, P. (2009). Advances in geometric morphometrics. Evolutionary Biology, 36(2), 235-247.

Muñoz-Colmenero, M., Jeunen, G. J., Borrell, Y. J., Martinez, J. L., Turrero, P., \& Garcia-Vazquez, E. (2015). Response of top shell assemblages to cyclogenesis disturbances. A case study in the Bay of Biscay. Marine Environmental Research, 112, 2-10.

Nordsieck, F. (1968). Europäische Meeres-Gehäuseschnecken (Prosobranchia) Vom Eismeer Bis Kapverden Und Mittelmeer. Stuttgart: Gustrav Fischer Verlag.

Payraudeau, B. C. (1826). Catalogue Descriptif et Methodique Des Annelides et Des Mollusques de l'Ile de Corse, Avec Huit Planches Representant Quatre-Vingt-Huit Especes, Dont SoixanteHuit Nouvelles. Paris: Levrault.

Philippi, R. A. (1836-1844). Enumeratio molluscorum Siciliae cum viventium tum in tellure tertiaria fossilium, quae in itinere suo observavit (Vol. Vol. 1). Berlin: Schropp.

Philippi, R. A. (1846). Die Kreiselschnecken Oder Trochoideen, Trochus. In H. C. Küster (Ed.), Martini, F. H. W. \& Chemnitz, J. H.: Systematisches Conchylien-Cabinet (pp. 166-224-225). Nürnberg: Bauer \& Raspe.

Philippi, R. A. (1849). Centuria altera testaceorum novarum. Zeitschrift für Malakozoologie, 5(7), 109.

Risso, A. (1826). Histoire Naturelle Des Principales Productions de l'Europe Méridionale et Particulièrement de Celles Des Environs de Nice et Des Alpes Maritimes. Paris: Chez F.-G. Levrault, libraire.

Rohlf, F. J. (1998). On applications of geometric Morphometrics to studies of ontogeny and phylogeny. Systematic Biology, 47(1), 147-158 discussion 159-67.

Rohlf, F. J. (2005). tps relative warps version 1.42. Stony Brook: Department of Ecology and Evolution. New York: State University.

Rohlf, F. J. (2009). tps Utility program. Ecology and Evolution, SUNY at Stony Brook.

Rohlf, F. J. (2010). TPSDIG2. 16. Stony Brook, NY: Department of Ecology and Evolution. New York: State University.

Schrödl, M., \& Haszprunar, G. (2016). Do we need epitypes in zoology? Spixiana, 39(2), 199-201.

Silvestro, D., \& Michalak, I. (2011). raxmlGUI: A graphical front-end for RAxML. Organisms Diversity \& Evolution. https://doi.org/10.1007/ s13127-011-0056-0.

Thiele, J. (1929-1935). Handbuch der systematischen Weichtierkunde. Jena: Gustav Fischer Verlag.

Thompson, D. W. (1917). On growth and form. Cambridge: University Press.

Uribe, J. E., Williams, S. T., Templado, J., Buge, B., \& Zardoya, R. (2016). Phylogenetic relationships of Mediterranean and NorthEast Atlantic Cantharidinae and notes on Stomatellinae 
(Vetigastropoda: Trochidae). Molecular Phylogenetics and Evolution, 107, 64-79.

Weigand, A. M., Jochum, A., Slapnik, R., Schnitzler, J., Zarza, E., \& Klussmann-Kolb, A. (2013). Evolution of microgastropods (Ellobioidea, Carychiidae): integrating taxonomic, phylogenetic and evolutionary hypotheses. BMC Evolutionary Biology, 13, 18.

Williams, S. T. (2012). Advances in molecular systematics of the vetigastropod superfamily Trochoidea. Zoologica Scripta, 41(6), 571-595.

Williams, S. T., \& Ozawa, T. (2006). Molecular phylogeny suggests polyphyly of both the turban shells (family Turbinidae) and the superfamily Trochoidea (Mollusca: Vetigastropoda). Molecular Phylogenetics and Evolution, 39(1), 33-51.

Williams, S. T., Karube, S., \& Ozawa, T. (2008). Molecular systematics of Vetigastropoda: Trochidae, Turbinidae and Trochoidea redefined. Zoologica Scripta, 37(5), 483-506.

Williams, S. T., Donald, K. M., Spencer, H. G., \& Nakano, T. (2010). Molecular systematics of the marine gastropod families Trochidae and Calliostomatidae (Mollusca: Superfamily Trochoidea). Molecular Phylogenetics and Evolution, 54(3), 783-809. 